NIST Technical Note 2173

\title{
Validation Capabilities of the NIST Campus Power System to Evaluate Distributed Control of Grid-Edge Distributed Energy Resources
}

D. M. Anand

C. Freiheit

T. H. Chen

M. A. Ehsan

T. C. Linn 
NIST Technical Note 2173

\title{
Validation Capabilities of the NIST Campus Power System to Evaluate Distributed Control of Grid-Edge Distributed Energy Resources
}

\author{
D. M. Anand \\ C. Freihert \\ M. A. Ehsa \\ T. C. Linn \\ Smart Grid and Cyber-Physical Systems Program Office \\ Engineering Laboratory \\ T. H. Chen \\ Data, Security and Technology Group \\ Engineering Laboratory
}

This publication is available free of charge from:

https://doi.org/10.6028/NIST.TN.2173

August 2021

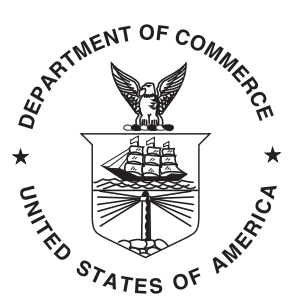

U.S. Department of Commerce Gina M. Raimondo, Secretary

National Institute of Standards and Technology James K. Olthoff, Performing the Non-Exclusive Functions and Duties of the Under Secretary of Commerce for Standards and Technology \& Director, National Institute of Standards and Technology 
Certain commercial entities, equipment or materials may be identified in this document in order to describe an experimental procedure or concept adequately. Such identification is not intended to imply recommendation or endorsement by the National Institute of Standards and Technology, nor is it intended to imply that the entities, materials, or equipment are necessarily the best available for the purpose.

National Institute of Standards and Technology Technical Note 2173

Natl. Inst. Stand. Technol. Tech. Note 2173, 62 pages (August 2021) CODEN: NTNOEF

This publication is available free of charge from: https://doi.org/10.6028/NIST.TN.2173 


\begin{abstract}
This report outlines National Institute of Standards and Technology (NIST)'s validation tools developed to help address the smart grid's emergent needs and complement research and standards coordination efforts. Rapid advances have enabled an opportunity for the grid to autonomously and simultaneously control generation, balance load, and regulate power quality. The grid will need this coordinated control to regulate power and voltage in realtime, to meet fast-changing power demands, especially with increasing distributed energy resources. To do this, the grid needs measurements from across the distribution network to accurately assess demand and prevent negative impact of variable grid-edge generation. Additionally, such a coordinated control system depends on a new operating paradigm with two-way information flows between domains of the grid. The different operational domains and the information flows between them are outlined in the NIST Framework and Roadmap of Smart Grid Interoperability Standards, Release 4.0 [1].

The coordinated control mentioned above requires improved observability of the state of distributed resources and depends on marshalling increasingly decentralized control systems. To implement such a system and meet system level performance objectives, distribution system operators (DSOs) need an understanding of the bandwidth, security and integrity considerations while also ensuring reliability at the grid's edges. Industry is also concerned with how to represent uncertainties like latency and cyber-vulnerabilities in a complex cyber-physical system. Additionally, industry is concerned that standards are focused more on devices than on coordinated control systems.

Thus, this report presents an overview of a data acquistion and control system designed by NIST as a validation tool and deployed on the NIST campus in Gaithersburg, MD. This system is comprised of interoperating computing resources, cybersecurity infrastructure, communication links and data interfaces to both take measurements and to transmit control inputs to commonly used distribution system control components. An important proof of concept included in the aforementioned validation system is a data pipeline optimized to stream data from a $5 \mathrm{MW}$ PV inverter and multiple sources of synchronized phasor data. This pipeline utilizes industry standard information modeling and communication standards with the intent of being compatible with industry standard testing equipment. This capability demonstrates how DSOs could test current standards against functionality - such as phasor based control of distribution circuits.
\end{abstract}

\title{
Assumptions and Assessment of Needs
}

The research presented in this report is motivated by these assumptions:

1. The grid is evolving towards coordinated control of distributed energy resources.

2. Formal methods to quantify uncertainty in power systems will be standardized across the DSO community. 
3. Industry standards and testing protocols will evolve to capture system-level performance.

An assessment of the current situation indicates that it is computationally infeasible to fully model and centralize the control of distribution system operations, especially given the uncertainties associated with distributed generation. However, it may be possible to improve computational feasibility using approximation tools such as reachability analysis for planning and modeling. Recent state-of-the-art methods may enable coordinated control of distributed energy resources. These include optimization-based methods and consensus-based controls. Regarding standards and testing, NIST assesses that standards-setting organizations (SSOs) are in the early stages of working with industry to develop precise and accepted system-level interoperability test methodologies [2] and that these efforts will be positively impacted with the availability of validation and analysis tools tailored to distribution circuits.

\section{Areas of research and development for validation}

The NIST Smart Grid Program has initiated several research projects to address the gap in validation and test tools. These projects include efforts to harmonize information models to help DSOs integrate groups of distributed energy resources on their primary and secondary feeders into an existing Distribution Management System (DMS). Researchers at NIST are developing an Adaptive Reference Governor scheme to assist in distributed control of constrained distributed energy resources (DERs). This Reference Governor is a control strategy that predicts the evolution of the system state to enforce pre-specified constraints on the inputs, states or the outputs of a system. Additionally, NIST is using its expertise in electrical metrology and test design to develop software libraries, calibration methods and automated test software to meet validation needs for controlling distributed energy resources.

\section{Campus capabilities for validation}

This research is performed using the facilities of NIST campus in Gaithersburg, Maryland. These include a distribution network with four $13.8 \mathrm{kV}$ feeders that span the campus. In addition to a local power utility, this network is supplied by two large on-site, distributed generators in addition to four smaller generators. Nodal state variables such as voltage and current magnitude, real and reactive power are reported to a central database, along with some power quality measurements such as total harmonic distortion, frequency, and power factor. Measurements are acquired at terminal nodes.

A $400 \mathrm{Mbps}$ data pipeline for inverter and phasor data was implemented to meet the needs of emerging research needs. The pipeline captures time-aligned data from multiple sites enabling researchers to monitor and respond to fast occurring events. NIST researchers have used the capabilities of the streaming pipeline to validate an AdaptiveContractive Reference Governor for constraint aware fast responding power converters. 


\section{Key words}

Distributed Energy Resources; Smart Grid; Distributed Control; Validation; Power Systems; PV generators; Hardware in the Loop; Reference Governor. 


\section{Table of Contents}

1 Introduction 1

1.1 Purpose 1

1.2 The Rapidly Evolving Electrical Energy Infrastructure 1

1.3 Fast Changes in Power Generation 1

1.4 Improved Monitoring for Situational Awareness 2

1.5 Realizing a New Operating Paradigm 2

1.6 Coordinating Across the Grid 2

1.7 Industry Concern- How to Represent Uncertainty in Realistic Operating Conditions 4

1.8 Industry Concern-Standards Focused on Devices Rather than Coordinated Control Systems 4

2 Assumptions and Assessment of Needs 5

2.1 Assumptions 5

2.2 Assessment of Uncertainty in Power Systems 5

2.3 Assessment of Coordinated Control of DERs 8

$\begin{array}{lr}2.4 \text { Standards and Testing } & 10\end{array}$

3 Campus Capabilities for Validation $\quad 11$

3.1 The NIST Distribution Network $\quad 12$

3.2 Data Pipeline 13

3.2.1 Stream Processing System Overview 15

3.2.2 Technologies and Features 17

3.3 Communication Infrastructure 20

3.4 Grid-edge Computing Test Platform 22

3.4.1 Adaptive Reference Governor for Grid-tied Photovoltaic Inverters 25

3.5 Campus in the Loop Experiments 30

4 Areas of Research and Development for Validation 32

4.1 Information Model Harmonization 34

4.2 Composition and Constraint Enforcement 37

4.3 Validation of the Information Exchange Required for DER Control 43

$\begin{array}{ll}\text { References } & 47\end{array}$

\section{List of Tables}

Table 1 Table showing salient features of the four classes of wireless technologies used on the NIST campus to support validation efforts.

Table 2 Configurable parameters for the Volt-Watt function as specified in the IEEE 1547 Standard. Per unit voltage values are normalized to nominal grid voltage $V_{\text {nominal }}$. 
Table 3 A mapping between data objects in the IEC 61850-7-4 and IEC 61850-7-420 standard against parameters required for implementation of the IEEE 1547 Volt-Watt function.

\section{List of Figures}

Fig. 1 An illustration of the cyber-physical interconnections that underpin the smart grid.

Fig. 2 A schematic diagram showing inter-operating stakeholders in a power system with communication pathways.

Fig. 3 A schematic showing the campus components on the NIST campus that have been included to serve as validation tools for a generic distribution system.

Fig. 4 Annotated diagram of a near future, high-DER distribution circuit.

Fig. 5 Data Pipeline designed to collect streaming data from multiple data sources on campus using the Confluent Kafka stream messaging platform.

Fig. 6 Real and Reactive power output over 96 hours, sampled at 10 samples per second.

Fig. 7 Scatter plot showing real power vs. reactive power values over a range of operating conditions.

Fig. 8 Scatter plot showing voltage vs. reactive power values over a range of operating conditions.

Fig. 9 Scatter plot showing voltage vs. active power values over a range of operating conditions.

Fig. 10 A screenshot of a monitoring dashboard displaying the live behavior of one PV generator system.

Fig. 11 A comparison of latency measurements for three high speed communication links. Subfigures a, b and c correspond to WLAN, P2P and wired Ethernet links respectively.

Fig. 12 A schematic diagram showing the key components of the NIST developed test platform for grid-edge intelligence. Image of the TWB-2945 fanless computer is used with permission.

Fig. 13 RG-AC governed Detailed Model of a $100 \mathrm{~kW}$ Grid-Connected PV Array in Simulink. Some of the blocks shown in this diagram are derived from a model in Mathworks' Simscape Electrical library.

Fig. 14 Detailed view of the VSC Controller block from Figure 13 showing the addition of the Reference Governor.

Fig. 15 Linear parameter-varying PV inverter model compared to simulated output. Positive power corresponds to power being delivered to the grid.

Fig. 16 RG-AC governed PV inverter test results. 
Fig. 17 A scatter plot showing line to line voltage variation observed at one of the campus PV generator sites measured over the full Volt-Watt control range. Two sets of scattered points shown on each plot correspond to $V_{C A}$ versus $V_{B C}$, and $V_{C A}$ versus $V_{A B}$ respectively.

Fig. 18 A scatter plot showing line to line voltage variation assumed in a simulated PV generator over the full Volt-Watt control range. Two sets of scattered points shown on each plot correspond to $V_{C A}$ versus $V_{B C}$, and $V_{C A}$ versus $V_{A B}$ respectively.

Fig. 19 DER models with corresponding DER Unit controllers simulated in realtime using $1 \mathrm{~ms}$ timesteps and 10ms control loops.

Fig. 20 Simscape Electrical model of 60 DERs forming proposed experimental architecture as per the schematic described in Figure 19.

Fig. 21 A schematic diagram showing the interacting elements in the validation environment.

Fig. 22 A plot showing network performance metrics for an IEC 61850 based distribution control system.

Fig. 23 DER tracking performance in response to feeder set points in the presence of network delays.

Fig. 24 DER tracking dynamics in response to feeder set point changes.

Fig. 25 Characteristic curves for Volt-Watt control of a DER as specified in the IEEE 1547 Standard.

Fig. 26 Class model for a generic IEC 61850 DER showing the four major classes of asset information.

Fig. 27 Inheritance diagram for the IEC 61850-7-420 information model of a distributed generator (DGEN).

Fig. 28 A schematic diagram showing a generic DER controller and some of the several information flows that would be needed to implement core functions. $\quad 40$

Fig. 29 Block diagram of RG governed system.

Fig. 30 Block diagram of RG-AC governed system.

Fig. 31 A NIST developed hardware-in-the-loop test setup used to evaluate implementation of the IEC 61850-9-3 time transfer standard.

Fig. 32 Figure shows the calibration fixture and illustrative test results from a power hardware-in-the-loop validation system. 


\section{Introduction}

\subsection{Purpose}

This report outlines the validation tools that NIST is developing to complement research and standards coordination efforts needed to advance the smart grid. These validation tools leverage components of the NIST campus electrical network. This section addresses the smart grid's emergent needs which NIST's validation tools are intended to help address.

\subsection{The Rapidly Evolving Electrical Energy Infrastructure}

The distribution grid infrastructure is becoming a system of decentralized generation, tightly coordinated control, autonomous fault recovery and embedded optimization capabilities. Its evolution accelerated over the last decade largely because of: increased use of nontraditional energy resources [3]; decline in the cost of photovoltaics (PV); and natural gasbased generation. At the same time, advances in power electronics and the availability of real-time computation have opened a new frontier of autonomous energy systems; these simultaneously control generation, balance load, and regulate power quality.

Much of this innovation is focused on the electrical distribution system. DSOs in several states, including Hawaii, California and Vermont, have provided a platform for increased distributed generation using coordinated sensing, measurement, and control of devices across their distribution grid [4-9].

\subsection{Fast Changes in Power Generation}

Distributed generation from PV systems provides an opportunity to compensate for fast changes in generation capacity (e.g. reduction in PV output during cloud-cover) using coordinated control systems. Without appropriate compensation, changes to generator injections at a rate comparable to the natural dynamics of the distribution circuit result in transient excursions in feeder voltage [10]. Compensation for voltage changes is typically provided by employing fast-acting mechanical tap-changing transformers, but frequent or continuous mechanical reconfiguration of tap-changing transformers greatly reduces their service life. Switching power electronics has been shown to reliably control voltage at their grid tie point using real-time control of their reactive power injection [11] and thus present an attractive alternative to mechanical tap-changing. The use of installed electronic power converters as a power conditioning resource has been widely studied in technical literature $[12,13]$ and carries the benefit of not only boosting their economic value to the installer but also providing multivariate control opportunities to the DSO.

During sunny days when PV systems peak, DSOs may experience reversed power flows. Bidirectional flow means that coordinated control requires more measurements from across the distribution network and higher sampling rates. These are needed to ensure the estimates of the system state are accurate and do not negatively impact core distribution optimization and protection schemes. 


\subsection{Improved Monitoring for Situational Awareness}

Trade-offs must be considered when implementing a coordinated control system. Its distributed sensing, data bandwidth, measurement robustness and network security are frequently cited as being the foremost concerns[14, 15]. However, it is possible to find practical successes in distribution circuit monitoring systems that are already deployed. One example is the large-scale implementation of Advanced Metering Infrastructure (AMI) in many parts of the United States and the measurable impact it has had on power quality [16]. AMI improves the DSO's situational awareness and permits two-way communication between the DSO and consumers. ${ }^{1}$ The successes and challenges of AMI deployment spread across a range of areas including developing standards, validation tools and testing protocols. Similar challenges and opportunities currently present themselves to the community as they aim to deploy technologies to coordinate and control distributed energy resources.

\subsection{Realizing a New Operating Paradigm}

A new operating paradigm for DSOs would involve designing for uncertainty using controllable variability (sometimes referred to as resource flexibility) [17]. In a truly distributed sense, the effects of uncertain or rapidly varying net-load (and voltage profiles) could be mitigated in real-time by distributed energy resources (DERs) such as PV inverters without the need for closed-loop central dispatch from the DSO. This delegation of real-time regulating tasks to grid-edge devices still has to be performed in a coordinated manner. In this new operating paradigm, the DSO would coordinate the flexibility of DERs instead of controlling them in real time which provides obvious benefits with regards to scalability and system complexity. The grid-edge control paradigm is expected to leverage AMI while expanding the communication and sensing infrastructure to actuators (i.e., inverters).

Figure 1 illustrates one vision of this new operating paradigm for the entire electric power system as presented in the NIST Smart Grid Framework 4.0 [1]. The figure illustrates the myriad of information flows between domains in the power system to achieve the level of coordination expected for a "smart grid".

\subsection{Coordinating Across the Grid}

The Smart Grid Framework concludes that system stakeholders must develop methods to effectively coordinate DERs and delegate some control authority to the edge of the grid in order to scale control systems with high DER penetration.

Figure 2 illustrates a grid-edge control scenario. It shows that most domains in a power system have a combination of distributed resources. From the perspective of a DSO, Figure 2 also shows that a typical distribution utility edge device is a smart meter or inverter

\footnotetext{
${ }^{1}$ Today's AMI samples customer loads every 15 minutes to 60 minutes and has large communication delays that prevent effective control. Yet, the AMI is capable of much faster sampling rates, and communication upgrades will greatly reduce delays.
} 


\section{Smart Grid Conceptual Model}

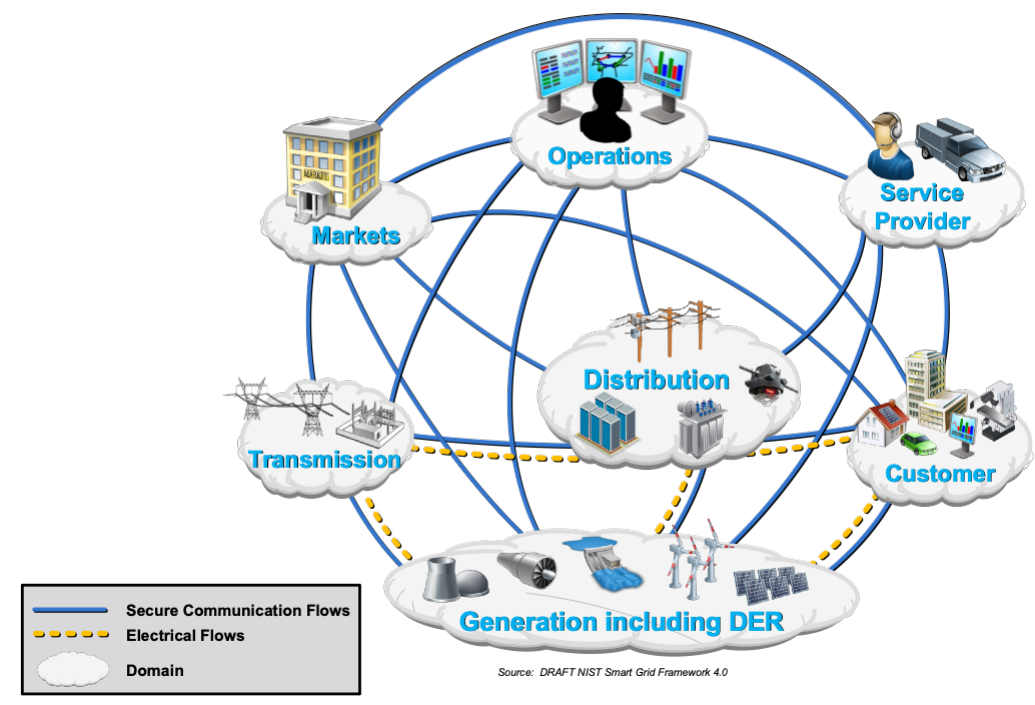

Fig. 1. An illustration of the cyber-physical interconnections that underpin the smart grid.

with distributed control assets, providing microgrid control or virtual battery/storage functions.

Equally important, this diagram illustrates parallel communications infrastructures. Operational communications, shown as a shared bus in the diagram, would realistically include multiple proprietary protocols and communication links. Phasor Measurement Units and smart meters may need to report measurements to a Microgrid Controller but would likely use parallel communication infrastructures to do so. The Microgrid Controller in turn may need to obtain set points and reference commands from the Distribution Management System. The validation problem for a DSO would be understanding the practical bandwidth, security and integrity considerations for all possible implementations of the scenario, shown in Figure 2, while still ensuring adequate performance of an inverter coordination scheme at the edge of the system.

The literature on coordinating inverters for fast power control in distribution systems has grown tremendously in the past decade. Most coordination algorithms are tested using simplifying assumptions; yet, much less published work exists on the considerations for implementing these algorithms. Consequently, utilities have a limited understanding of the value of the proposed control schemes and the effects of simplified models and system assumptions on robustness and stability. A need exists to provide performance guarantees on coordinated inverter-based control schemes under realistic operating conditions to increase trust in this technology. 


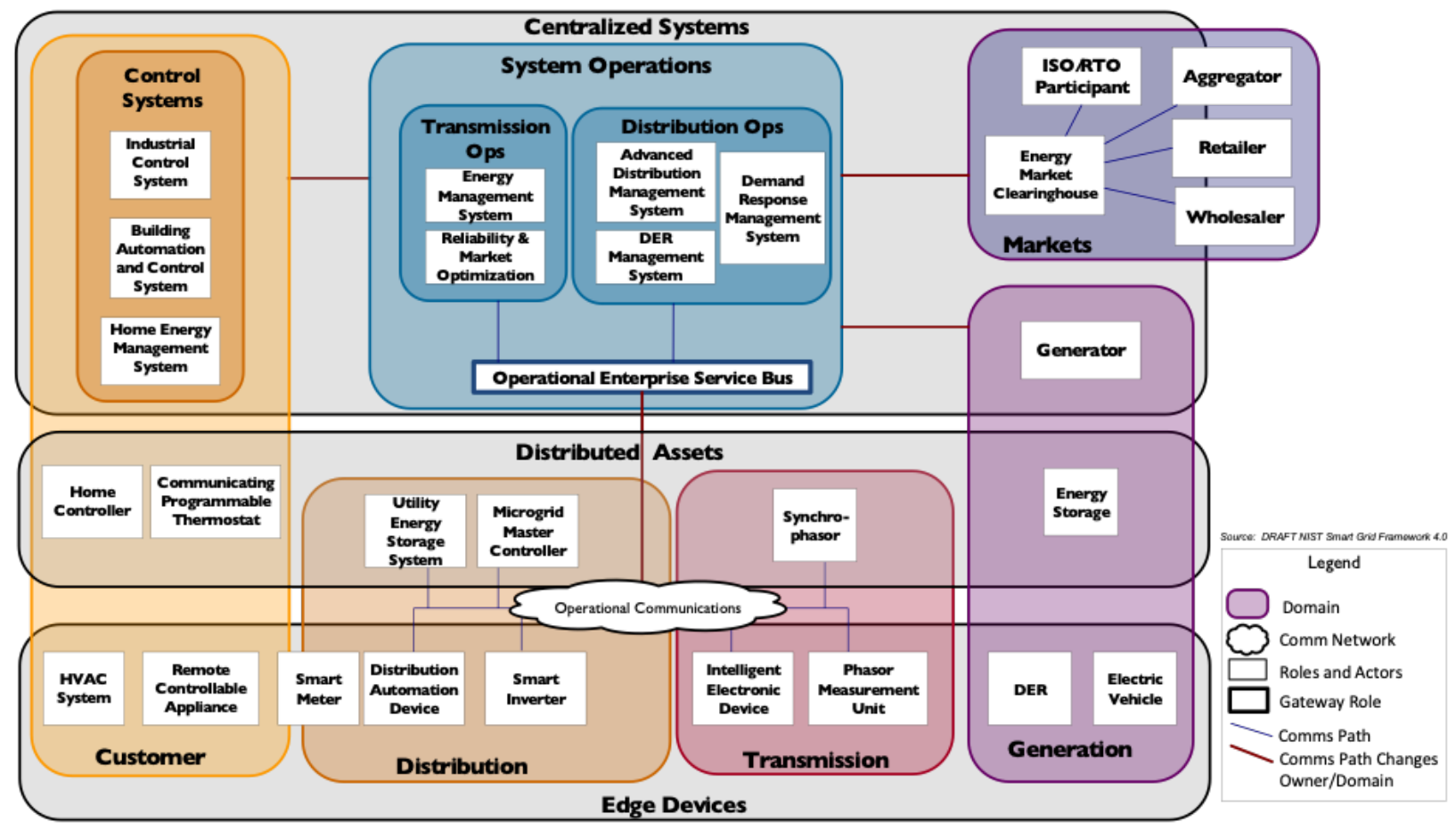

Fig. 2. A schematic diagram showing inter-operating stakeholders in a power system with communication pathways.

\subsection{Industry Concern- How to Represent Uncertainty in Realistic Operating Con- ditions}

To aid in their implementation of coordinated control systems for regulating a network of PV inverters, DSOs would benefit from an integrated approach to evaluate the impact of both cyber risks [18] and physical uncertainty [19, 20]. Characterizing the uncertainties in a complex cyber-physical system as in Figure 1 is especially challenging since cyber and physical domains are tightly interconnected [21]. Generally, uncertainity analysis present two classes of uncertainties:

- Implementation-specific aleatory variability in the process or system response, including communication latency, cyber vulnerabilities [22], device heterogeneity, etc.

- Epistemic uncertainty associated with incomplete state information [23], modeling gaps, measurement error, etc .

\subsection{Industry Concern-Standards Focused on Devices Rather than Coordinated Con- trol Systems}

Currently, a technical gap exists between methods for coordinated control of DERs (e.g., PV inverter or battery devices) and the metrics published in industry standards. Testing 
and certification requirements for advanced inverter functionality focus on single-device tests rather than interoperability and information sharing required for decentralized operation [24]. Documentary standards addressing performance metrics at the system-level are an essential step to fully realizing the salient effects of PV inverter-based distribution circuits [25]. Due to a standards gap, early PV inverter requirements forced unity powerfactor operation which neglected ancillary services from inverters to the grid. Even today's device-level standard IEEE 1547 only indirectly considers the power quality of the grid by providing some flexibility in disconnection and ramp-up timing [26]. However, the role of inverter heterogeneity and communication schemes for coordinated control are still not part of the standard [27]. The DSO community has expressed interest in suitable systemlevel frameworks that outline information sharing and control performance in systems with multiple inverters [9]. DSOs identified the following gaps:

1. Guidance on developing new system-level standards for inverter-based control schemes.

2. Improved metrics for distribution system reliability, power quality, and system security through robust regulation of terminal node voltages, power injections, and minimization of line losses.

3. Creation of a hardware-in-the-loop testing framework that extends to more general control topics, such as synthetic inertia, distributed line loss minimization and demand response.

4. Formulation of control theoretic problems that explicitly consider the effects of cyber and physical uncertainty.

\section{Assumptions and Assessment of Needs}

\subsection{Assumptions}

NIST's research and development for the grid is based on three assumptions:

1. That the grid is evolving towards coordinated control of distributed energy resources;

2. That formal methods to quantify uncertainty in power systems will be standardized across the DSO community;

3. That industry standards and testing protocols will evolve to capture system-level performance.

\subsection{Assessment of Uncertainty in Power Systems}

Accuracy of the system models is very important. Analysis of power system dynamical behavior is largely model-based. Actual system behavior is inferred from the simulated response of the system models. Even planning and operating decisions are influenced by 
the simulated system behavior. However, many model parameters (e.g., loads, renewable energy injections) can never be known with absolute certainty.

Closed-loop control schemes for the bulk energy system frequently employ a convexified power flow model. This is a computational simplification that is assumed to be accurate under most nominal operating modes.

For example, in the work presented in [28-30], thermal load on transmission lines is alleviated using coordinated control of storage and PV assets. In this work, the model errors introduced from convexification are mostly within $10 \%$.

However, the closed-loop response was unable to completely eliminate line temperature overloads. That is, feedback was unable to robustly reject disturbances from model inaccuracy. Robust performance in such cases is only restored by applying conservative constraints and limiting the control system to sub-par closed-loop performance [31].

In fact, in operational power networks, the effect of uncertainty is often not explored in the context of closed-loop control schemes, as the negative feedback loop is often assumed to reject a wide range of disturbances (including model inaccuracies and communication latency). In addition, the promulgation of distributed generation and variability of response in net-load assets (e.g., inverters) add further complications to closed-loop performance.

The control systems literature uses a formal notation that combines differential and switched-algebraic equations to describe large complex cyber-physical systems. This notation is called a hybrid dynamical system $[32,33]$. Using this approach, model parameters and inputs of complex systems that are unknown or approximated can be explicitly coded with uncertainty metrics. This means that when the actual system trajectory deviates from that of the model, state-feedback can be used to provide robustness against disturbances (e.g., input and parameter uncertainty). However, in employment of automatic control systems in critical infrastructures such as electrical systems, a-priori verification of safety and/or robustness is important to ensure adequate closed-loop performance. Monte Carlo analyses are the predominant approach used to study the impact of a variety of uncertainties in a hybrid dynamical system. Monte Carlo approaches are also commonly used to assess cyber vulnerabilities or communication loss over a range of outage scenarios.

However, it is computationally infeasible to use Monte Carlo analyses for complex models of power systems. This is due to:

- Underlying complexity and scale of the physical distribution system (e.g., large number of inputs and states);

- Mix of continuous and discrete states and inputs (e.g., tap-changing transformers);

- Limits on states and input;

- Role of communication systems (i.e., delays and topology); and

- Combinatorial scale of all possible system outcomes.

This complexity also increases with the addition of smart grid technologies, such as plug-in electric vehicles, PV inverters, and energy storage devices. 
One approach to improving computational feasibility is called trajectory sensitivity analysis, e.g., see [34-36]. It has been applied extensively to power systems [37-40]. Trajectory sensitivity analysis provides an accurate and computationally amenable method for approximating the trajectory of a hybrid dynamical system subject to a small perturbation resulting from one uncertain system parameter. This is achieved via linearization around a nominal trajectory. However, to provide guarantees in performance, one must verify all system trajectories which still require inefficient Monte Carlo techniques.

To overcome the need for exhaustively exploring all possible trajectories in an uncertain system, approximations are often employed to 'lump' or 'group' states, system parameters, and inputs. In such cases, trade-offs must be made between accuracy and computational performance [41]. There are many methods to group states based on likelihood, sensitivity, criticality or dependence.

This method of analysis is called reachability analysis. It is an attractive tool to explore and quantify the effects of uncertainty on closed-loop performance. Reachability analysis describes the set of states reachable from a set of initial states in finite time for hybrid dynamical systems. As such, reachability analysis is often used in hybrid systems for safety and robustness verification and can provide a guarantee of robustness against uncertainties [42].

However, the exact computation of reachable sets for general hybrid dynamical systems may still be computationally intractable [43]. Therefore, an approximation of the reachable set is often necessary to reduce the computational burden and provide useful insight into system and closed-loop performance.

To engender a useful approximation of the reachable set generally requires:

- Appropriate simplifying assumptions such as linear systems [41, 44, 45];

- Order-preserving dynamics [46]; or

- Convex bounds on actuation [47].

Under such conditions, the computational complexity of reachable sets can be greatly reduced and permit tractable implementations of external approximations (to verify robustness and safety) and internal approximations (to determine guaranteed performance bounds).

In the case of power systems described by nonlinear differential algebraic equations (DAEs), reachability analysis is often limited to small 1-2 bus systems [48]. For larger power systems, the reachable set can be efficiently approximated by ellipsoidal techniques for linearized system models $[49,50]$ and second-order Taylor-series approximations [51].

However, the resulting reach set is no longer an over-approximation of the exact reach set of the nonlinear system. This prevents formal guarantees of closed-loop performance for the underlying nonlinear system. To provide guarantees for the nonlinear power system, computations of reachable sets have been approximated efficiently by considering the set of linearization errors and application of convex polytopes (i.e., "zonotopes"). This is the 
set representation (instead of ellipsoids) [52, 53], which provides an attractive option for reachability analysis in uncertain power systems $[54,55]$.

From the perspective of validation, significant work is needed to identify the best suited simplifications for reachability analysis. Only theoretical reachability studies have been conducted on the coordinated control of inverters in a distribution system setting.

As a near term goal, the grid stakeholder community would get valuable feedback if hardware-in-the-loop testing of inverter control systems could be used to profile the reachable space of an actually deployed system. This is a better approach, compared to a linearized system, starting from the same initial states and reference set points (i.e., reactive power set points of inverters). Later, the uncertainty set should be expanded to include:

- Underlying nonlinear DAE system under communication delays;

- System parameters; and

- Measurement errors.

This report describes the data collection and tabulation infrastructure being developed to establish empirical bases for trajectory sensitivity maps and reachable spaces.

\subsection{Assessment of Coordinated Control of DERs}

Coordinated control of inverters in distribution systems is an attractive proposition [56, 57]. However, if the DSO communication or computation systems were compromised, all inverters would be left in the dark. Instead, a distributed architecture would be more reliable against communication outages and computational challenges. In fact, if consumers' inverters could communicate with each another and/or with the utility, the inverters could perform local computation and coordinate their decision-making.

Most inverters in a distribution circuit, though, operate behind the meter. Thus, a central entity such as a utility is unlikely to have access to operational information about the state, availability and control authority. This data barrier greatly limits effectiveness of centralized inverter control schemes. Furthermore, giving a central entity behind-the-meter awareness would shift the line between utility ownership and consumer ownership and require contested regulatory and market changes. In addition, the reliability of centralized schemes suffers from a concentration of risk at the central entity.

The most simple form of coordination is when no communication is needed and each agent cooperatively responds to its own local signals. This is considered a strictly local control law and is often called (fully) decentralized control. However, coordinated control employs direct or indirect communication between agents and is often formulated as a variant of a duality-based distributed optimization or consensus-based methods. The key factors differentiating literature on coordinated control involve the communication network (e.g., centralized, neighbor-to-neighbor, decentralized, and a/synchronous), grid topology (e.g., meshed vs. radial), and system assumptions (e.g., balanced vs. unbalanced system, steady-state vs. dynamic power flow, uniform line impedances). 
The following are recent state-of-the-art methods enabling coordinated control of DER:

Optimization-based methods: These are popular in distributed control due to the ability to take into account constraints on inputs and states and the availability of numerically robust solvers. As such, centralized optimization problems have been formulated for a system of inverters, and large-scale simulations provided insights into the development of simple local control laws that can recover up to $95 \%$ of centralized performance $[58,59]$.

Classical duality-based techniques can be applied to convert the central problem into many smaller distributed control problems. Through communication of dual variables, optimal (centralized) performance can be provably recovered after infinite communication iterations, which means that one cannot guarantee primal feasibility in actual implementation.

Therefore, robust optimization techniques are often used for distributed control as in [31]. A convex relaxation combined with the dual ascent algorithm is presented in [60] to investigate reactive power control under the assumption of uni-directional flows. Combining dual communication instances with actuation results in online distributed control, which provides promising synchronous and asynchronous real-time implementation avenues but suffers from non-trivial primal feasibility violations [61].

Consensus-based methods: These provide a framework for leaderless optimizations where agents exchange information over a pre-defined (possibly overlapping) communication topology to reach agreement (i.e., consensus) on a common decision [62]. The authors in [63], focus on steady-state voltage and reactive power control of inverters in distribution systems. The coordination is achieved via randomized gossip algorithms with overlapping communication networks. The inverters send local voltage and injection measurements to decentralized control points on the communications network which uses that information along with local grid topology information to rapidly converge on the minimal loss solution.

An unbalanced distribution model with consensus methods for coordination is investigated in [64]. A special case of consensus (which is an averaging problem) [65-68] provides a comprehensive distributed PI-control framework for frequency regulation in microgrid systems. The framework outlines a plug-and-play paradigm wherein distributed energy resources can enter or leave the control space without the need to modify control parameters. A combination of voltage and frequency regulation via droop-based control is proposed in [69].

The authors in [63] focused on steady-state voltage and reactive power control of inverters in microgrids and radial distribution systems. The coordination is achieved via overlapping communication networks (i.e. clusters) without the need for a centralized coordinating entity. The inverters send their communication cluster local voltage and injection measurements, and clusters use that information along with local topology information to rapidly converge (in 40 to 60 iterations) on the minimal loss solution.

The work in [61] considers online duality-based coordination under synchronous and asynchronous communication schemes between neighboring inverters. A dual decomposition/ascent algorithm is presented that has inverters actually actuate the system at every 
iteration by updating the reactive power injection set point. Since a duality approach underlies the proposed method, voltage may exceed limits during iterations.

Using a hierarchical scheme to coordinate conventional controllers, the authors in [65] employ distributed droop and PI control of inverters for frequency control in a microgrid setting. This is achieved by combining the hierarchical droop and integral control (common in dynamic power systems) into distributed 'averaging' PI control (i.e., dynamic concensus) that recovers original operating points. The analysis of interdependent control loops in power systems lends itself to plug-and-play coordinated control of inverters in microgrids.

Addressing the multivariate nature power systems control, the authors in [69] consider the problem of simultaneous coordinated V-f, P-Q, and MPPT control of a PV inverter with battery storage. This work focuses on an islanded microgrid setting with a single large PV system and battery storage. The objective is to satisfy voltage, frequency, storage, and power flow constraints, and this is done through multiple PID feedback loops.

As this list of research efforts illustrates, there is variety and sophistication in the choice of control algorithms for a range of inverter- based functions. One of the limiting factors is the determination of an appropriate delegation structure that retains analytical consistency while providing insight into system uncertainty and closed loop performance.

From a validation point of view, it would be valuable to develop a delegation architecture that supports different control algorithms at the device level, while at the same time providing a consistent abstract interface to coordinators and other subsystems. This abstraction should also be compatible with the geometric approximations required for reachability analysis described in the previous subsection.

This report highlights a set-theoretic formulation of both control objectives and uncertainty. As an example of implementation, a reference governor is designed for a PV system that is agnostic to the control algorithm of choice; yet, it addresses the concerns of providing enforceable and composable constraints to local controllers so that the delegation of control objectives and the abstraction of robustness margins can be evaluated against real data and hardware.

\subsection{Standards and Testing}

The increasing penetration of PV systems (esp. in CA, HI, and VT) has led to growing concerns among DSOs about the impact of distributed generation on the distribution grid [9]. In response, IEEE standard 1547 was updated to provide performance requirements on advanced control and communication functionalities between inverters (and other distributed energy resources) and with the DSO [70]. The certification test procedure, UL 1741, has been re-developed to supplement IEEE 1547 to ensure safe operation of equipment associated with distributed energy resources [26]. In addition, IEC 62786 provides technical requirements for planning, design, operation, and connection of DERs to the distribution grid [71].

Together with IEEE standard 2030, industry has published interoperability guidelines for the integration of power, communication, and information technologies [72]. Inverters 
manufactured today have advanced control and communication functionalities that permit DSOs to move away from constant power factor control of inverters. These functions consider coordinated control of distributed energy resources (e.g., dynamic reactive power control for Volt-VAr regulation) [25].

To reliably implement coordinated control of PV inverters, the DSO must first perform impact studies to obtain a clear understanding of system dynamic performance, including expected levels of current from PV inverters and their response to grid disturbances. However, DSOs do not have access to manufacturers' detailed models of inverter design and internal control schemes and have to rely on test results provided by certification agencies (e.g., based on the UL-1741 method).

Unfortunately, in current practice, certification focuses on device performance rather than system-level operation [73]. Indeed, certification testing practices neglect:

- System-level effects of the grid (e.g., voltage and impedance levels);

- Proximity of the device to conventional control devices (e.g., capacitor banks);

- Impact of nearby inverter actuation, performance of communication schemes (e.g., delays) [9]; and

- Gaps in information mapping between device and system level object models [74].

Even though PV system penetration is increasing rapidly, industry standards committees are only in the early stages of working with industry to develop precise and widely accepted system-level, interoperability testing methodologies.

As such, there is an immediate need to develop procedures that can capture the complex effects of multiple inverters with diverse data interfaces acting in a coordinated fashion on a distribution system. The need for such methods is exemplified by the rise of real-time hardware-in-the-loop (HiL) testing as a cost-effective means to investigate system-level interactions [9].

As an example, a DSO may be interested in evaluating if inverter-based conservation voltage reduction (CVR) [75, 76] can be achieved using current IEEE 1541 and IEEE 2030 inverter control standards. This test will require an HiL experiment with all of the interfaces, controllers and information models appropriate for existing standards while also providing the ability to implement a simulated CVR function; this is the type of test case for which the validation tools described in this report are suited. It is our expectation that vetted validation tools will help identify specific modifications to future standards.

\section{Campus Capabilities for Validation}

This section outlines the work being done to leverage the electrical power system on the NIST campus in Gaithersburg, MD, as a validation testbed. This section also presents validation experiments which have used campus resources to address industry concerns and to test modeling, simulation, control and communication research projects summarized in Section 4. 


\subsection{The NIST Distribution Network}

NIST's network is unique in that it employs four $13.8 \mathrm{kV}$ distribution feeders that span the campus. This distribution network is supplied by at least three large on-site, distributed PV generators in addition to the local power utility.

Terminal nodes primarily operate as loads on this network. Some terminal nodes have small distributed generation assets attached. However, those assets are restricted from feeding into the distribution network to ensure radial power flow. Each node is comprised of step-down distribution transformers, providing secondary (480Y277V) service to racked switchgear, breakers and monitoring equipment. Additional service transformers within each terminal node provide 208 Y120V service for offices and lighting loads.

In the last several years, most of the terminal nodes have been upgraded with networked monitoring equipment which report quasi-static measurements of each $13.8 \mathrm{kV}$ primary feeder and loads at the secondary feeder level. Nodal state variables such as voltage, current, real power and reactive power are reported to a central database, along with a few power quality measurements such as total harmonic distortion, frequency, and power factor. The reported values are acquired over a multi-second aperture and are adequate to provide minute-scale $(60 \mathrm{~s})$ nodal snapshots of network state.

The topology of the campus electrical system is similar in structure to a section of an archetypal residential distribution system. The campus system has a trunk and branch radial structure with sectionalizing breakers, a single head end sub-station and tap changing transformer-based voltage support (see Figure 3). The campus circuit has many more measurement points than a standard distribution circuit, but this level of instrumentation may not be very far away in the future, especially for distribution circuits with high DER penetration.

In addition to the terminal loads, the campus electrical system also hosts one 5 MVA PV generator, two $250 \mathrm{kVA}$ PV generators, and one $70 \mathrm{kVA}$ generator. All four systems are instrumented with measurements at approximately one measurement per second period.

A research grade $30 \mathrm{kVA}$ bifacial array is currently being installed with a IEEE 1547 compliant grid service inverter and Phasor Measurement Units at the point of coupling on the secondary feeder. Figure 3 illustrates the two types of DERs available on campus, i.e., distributed PV generators and building loads. Both DER systems have primary and secondary interconnection points that are instrumented with voltage, current, power factor and frequency sensors. Many of the DERs on campus also have dedicated power quality and condition monitoring sensors on breakers and transformers.

The data collection infrastructure, either installed or being developed on the NIST campus, is well suited to test concepts and models in the context of a full scale distribution circuit, such as the one shown in Figure 4.

This diagram is a test case that is loosely based on a real circuit but is augmented with components to represent a future high DER operating environment. Versions of this circuit have been used to test algorithms focused on distribution system optimization and control [77]. The line thickness in the diagram could signify notional electrical distance from the head-end node or the nodal voltage magnitude. In traditional radial distribution circuits, 


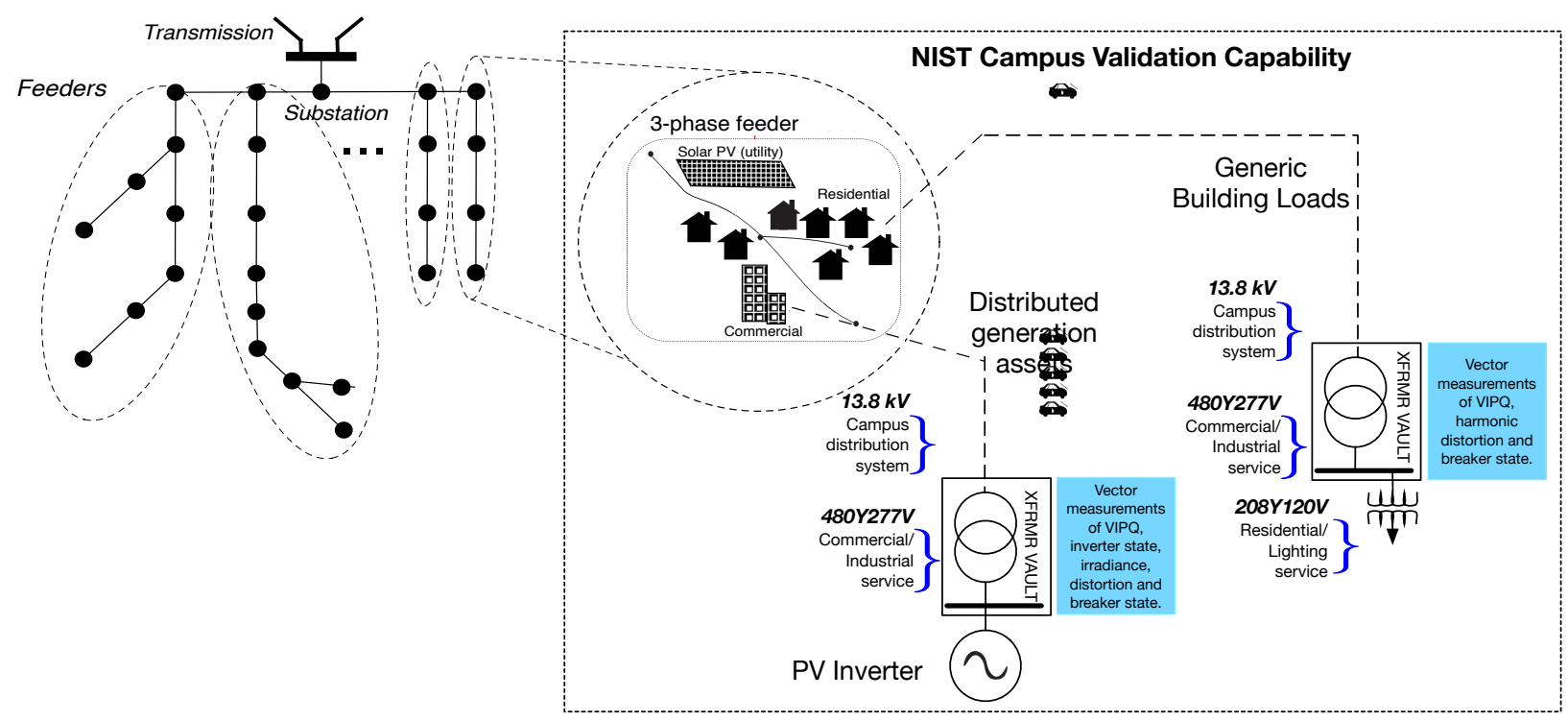

Fig. 3. A schematic showing the campus components on the NIST campus that have been included to serve as validation tools for a generic distribution system.

these two properties would be equivalent.

The circuit is radial in nature, reflected by monotonically decreasing voltage from headend, outwards. A small group of DER assets at the far end of one of the branches in the circuit is organized like a network or mesh. The circuit also features weakly connected nodes on lightly loaded networks that may operate in islanded or grid tied modes. The circuit is also assumed to be well endowed with metrology points and the required sensors. This assumption is made in order to consider the scalability of communication and information management systems that would be needed.

Lastly, the circuit has over a hundred DERs (represented by blue dots in Figure 4), distributed in all expected contexts across the network. Some of the DERs represent utilityowned assets, connected directly to the trunk of the circuit, while some others are connected to the edge of the circuit, representing roof-top or customer-owned generation assets.

\subsection{Data Pipeline}

As discussed in Section 1, one of the key validation concerns raised by industry is the evaluation of distributed systems. The tools used to calculate uncertainty, measure performance and estimate robustness must be updated to evaluate entire systems, rather than devices. Also, the systems being analyzed are frequently comprised of both cyber and physical components and communication links between them.

To address this concern, the NIST campus testbed project built a data pipeline infrastructure. It addresses the dual goals of collecting real data and measurements from assets on the NIST electrical network as well as providing the flexibility and software platforms to implement tools, algorithms and tests for research purposes. 


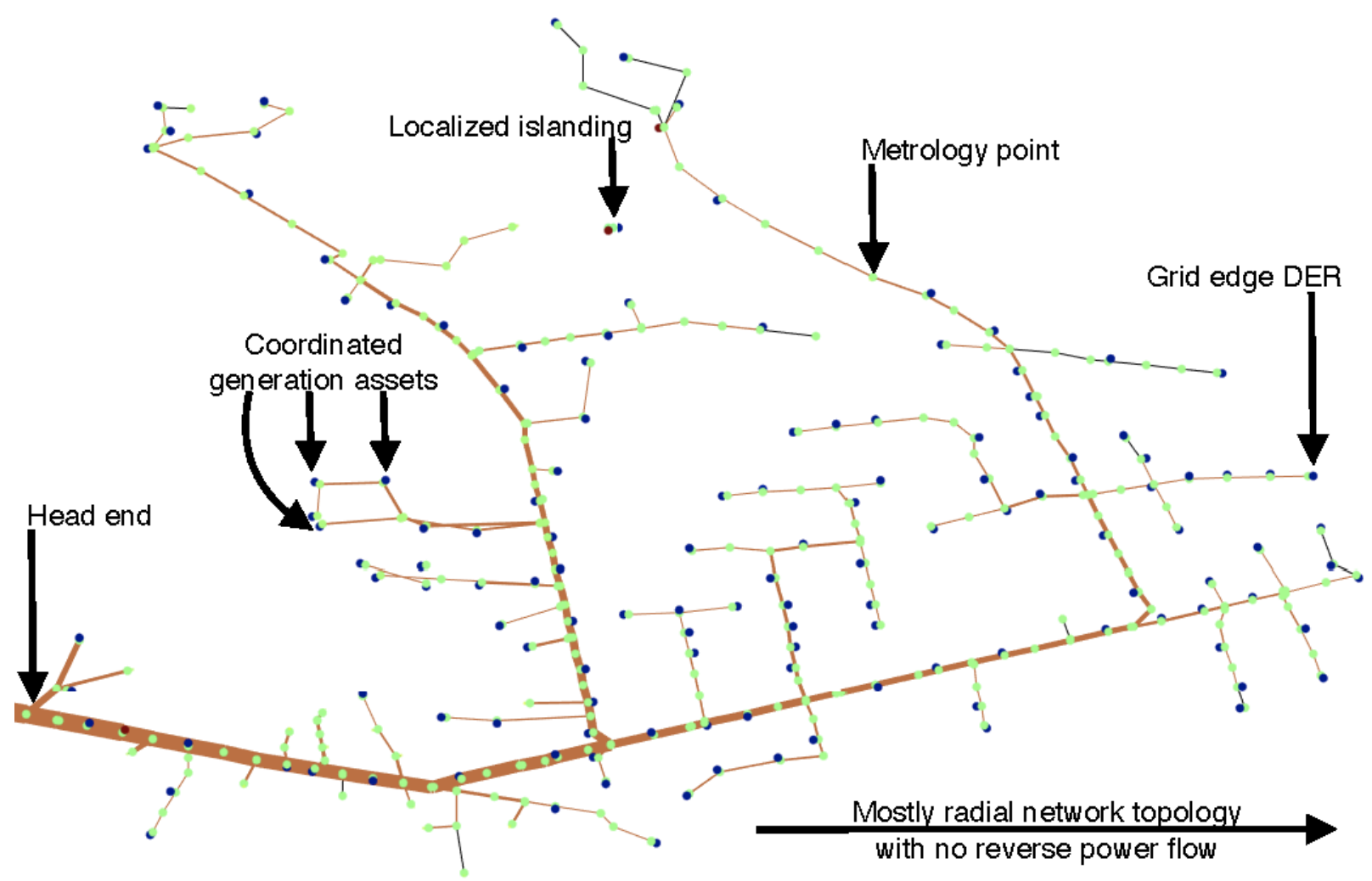

Fig. 4. Annotated diagram of a near future, high-DER distribution circuit. The validation tools described in this report were designed to address the control challenges for this prototypical circuit. 
Central to the data pipeline's design is stream processing of data. In stream processing, data are continuously processed as new data, which become available for analyzing. These data are processed sequentially as opposed to batched processing where data are first buffered and processed non-sequentially as bounded units. The key difference with regard to software design is that analytics on a batched system can only be periodically executed. In a stream processing pipeline, an unbounded stream may be pulled in by a 'listening' analytics system and processed continually.

This design choice has many software design implications for analysis and time synchronization between diverse data streams. However, it is an essential design choice for the pipeline to capture, format and store real-time data with as low a latency as possible and to trigger analysis actions immediately when relevant events arrive in the stream.

Confluent Kafka is the event streaming platform used for the data pipeline. It is based on the open-source Apache Kafka [78] that is used as the central publish-subscribe hub to our data processing. In this section, the desirable features of Kafka are described highlighting the specific technologies that were selected during deployment and how they were configured.

\subsubsection{Stream Processing System Overview}

Figure 5 illustrates how the data pipeline is used to stream disparate data generated at different rates. For instance, a Gateway Modbus server was installed at each PV generation site to relay metrics from multiple sources (weather, inverters, etc.): this server updated measurements every 5 seconds. For faster measurements, it is possible to pull data directly from manufacturers' Inverter Modbus servers which refresh every 100 milliseconds.

Downstream, a Serializing Producer fits those data into their own Avro Schemas and produces serialized message strings for their corresponding Kafka Topics (e.g., weather topic, inverter state topic). The Schema Registry comes into play when the producer separates schema and data so that schema can be reused and not transmitted with every data message. The schemas are further reused when the Sink Connector consumes and deserializes data, continuously, from the Kafka Topics before transposing them onto Postgres DB tables. The parallels in this design to the object-relational design of the IEC 61850 protocol for electrical power systems is notable.

Topics, as used in this design, can be thought of as logical nodes in the IEC 61850-7420 standard. The schema registry employed in the pipeline can be populated with IEC 61850 or another information model of choice, depending on the experimenter's use case. The deserializing step isolates the choice of communication information design from the database system.

Lastly, a Grafana Dashboard was implemented to query and aggregate time series from the Postgres tables and to visually monitor and graph these data in real time. Grafana is a popular dashboard design tool and can be used to build custom dashboards for a tailored experiment's specific needs. 


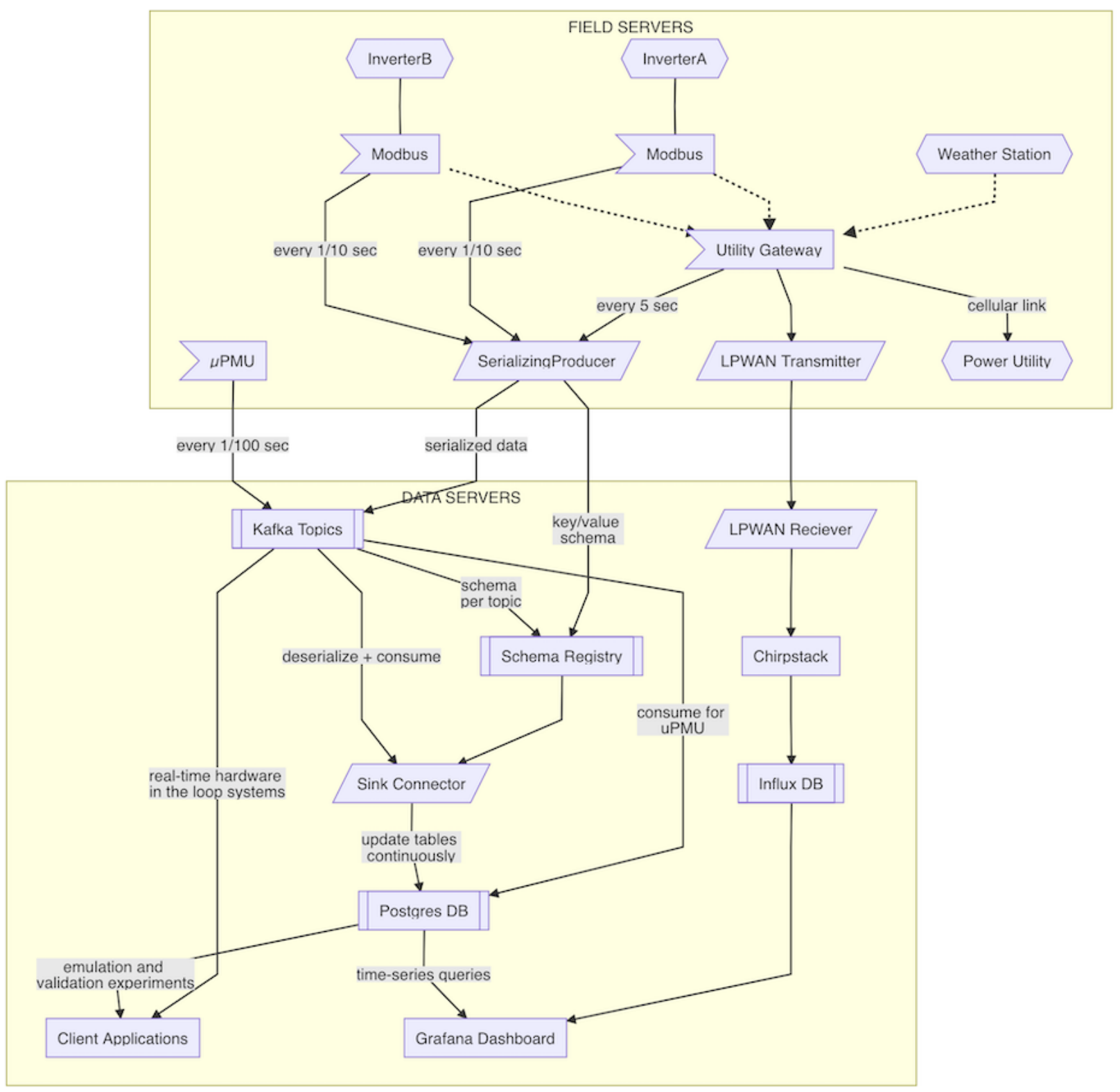

Fig. 5. Data Pipeline designed to collect streaming data from multiple data sources on campus using the Confluent Kafka stream messaging platform. 


\subsubsection{Technologies and Features}

- Apache Kafka ${ }^{2}$ : Kafka was selected to meet the speed, latency and bandwidth needs of all the data sources currently integrated into the campus instrumentation project while leaving capacity for future expansion of both the desired bandwidth and the number of parallel streaming sources. Kafka also provides a multi-producer,multiconsumer persistent log which serves as a buffer between data sources and downstream long-term storage. This feature is utilized if the database is experiencing delays in processing data or if the connection to the database is temporarily lost. Lastly, the log simplifies the challenges of coordinating software development between the producer and consumer. These two sides of the data flow path are only loosely coupled by a specified data schema (e.g., ApacheAvro) for logged messages. Besides this coupling, changes in a producer do not affect how a consumer is implemented and vice versa.

- Apache Avro ${ }^{3}$ : Kafka by itself does not enforce a particular data format. However, having a common data format and schemata is key to efficiently integrating multiple data sources in a pipeline. It is simpler for a developer to implement a lightweight, unambiguous interface that supports multiple programming languages rather than maintaining a multitude of one-off schema converters. The Apache Avro format was followed in this project to aid in implementation across multiple developers. The Avro schema was able to accommodate the data sources currently in use including; inverters, weather stations and PMUs.

- Confluent Schema Registry ${ }^{4}$ : The Schema Registry facilitates storage and retrieval of Avro schemas as part of producer-consumer (de-)serialization process. It is centralized so that producers and consumers can register and locate schemas relevant to data being handled. The separation of schema and data makes the encoded messages more compact and efficient for storage and transmission.

- Avro Serializing Producer ${ }^{5}$ : This is a high-level producer included as part of Confluent's open-source Python client (https://github.com/confluentinc/confluent-kafkapython). It wraps lower level APIs that handle calls to schema registry and Kafka topics so that the application developer only needed to ensure data fed into the message producer was compatible with a specified schema. Likewise, on the receiving

\footnotetext{
${ }^{2}$ Kafka Benchmarking tests

https://engineering.linkedin.com/kafka/benchmarking-apache-kafka-2-million-writes-second-three-cheapmachines

${ }^{3}$ Why Avro for Kafka Data?

https://www.confluent.io/blog/avro-kafka-data/

${ }^{4}$ Schema Management

https://docs.confluent.io/current/schema-registry/index.html

${ }^{5}$ Example avro_producer

https://github.com/confluentinc/confluent-kafka-python/blob/master/examples/avro_producer.py
} 


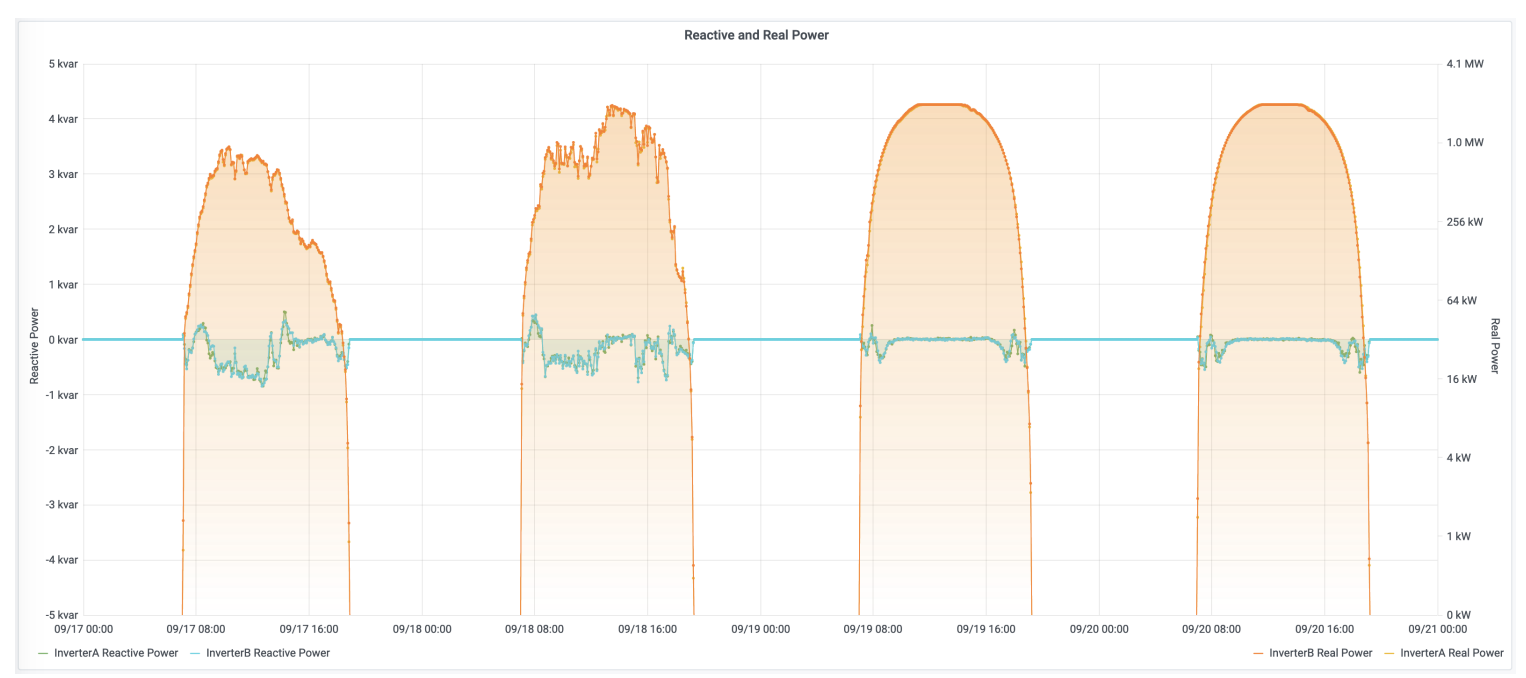

Fig. 6. Real and Reactive power output over 96 hours, sampled at 10 samples per second.

end, a consumer (e.g. Avro Deserializing Consumer) should be able to decode messages from the topics, as long as it can retrieve the corresponding Avro Schemas from a registry. On the other hand, in the unfortunate event that a producer does not use and register a schema, a programmer will need to learn how the producer encoded the messages before decoding messages more or less manually.

- Kafka Connect - JDBC Sink ${ }^{6}$ : For well-known data sources and destinations, like Postgres DB in our use case, Confluent platform provides reusable connectors. These are built on top of Kafka consumer and producer APIs that one can "plug-in" to automate the Extract-Transform-Load process. In the implementation described here, a Java Database Connectivity Sink connector was configured to subscribe to specific Kafka topics used. The connector decodes messages using Avro schemas from the Schema Registry, and inserts those time-series data into corresponding Postgres tables.

Tests of the data pipeline considered three major use cases: historical time series queries, correlation studies and real time monitoring. Figure 6 shows a query of real and reactive power sampled at 10 samples per second of a PV generator output over four days. This query has over 7 million data points. Fine data resolution over multiple days allows researchers to observe reactive power transients on cloudy days and also to observe voltage regulation effects as the generator powers on and powers off at sunrise and sunset respectively.

The use of Apache Kafka and Kafka topics allows correlation studies to be conducted on both live data and historical data. Figures 7, 8 and 9 show Watt-VAr, Volt-VAr and Volt-Watt correlation studies.

${ }^{6}$ Java Database Connectivity (JDBC) Sink Connector for Confluent Platform https://docs.confluent.io/current/connect/kafka-connect-jdbc/sink-connector/ 


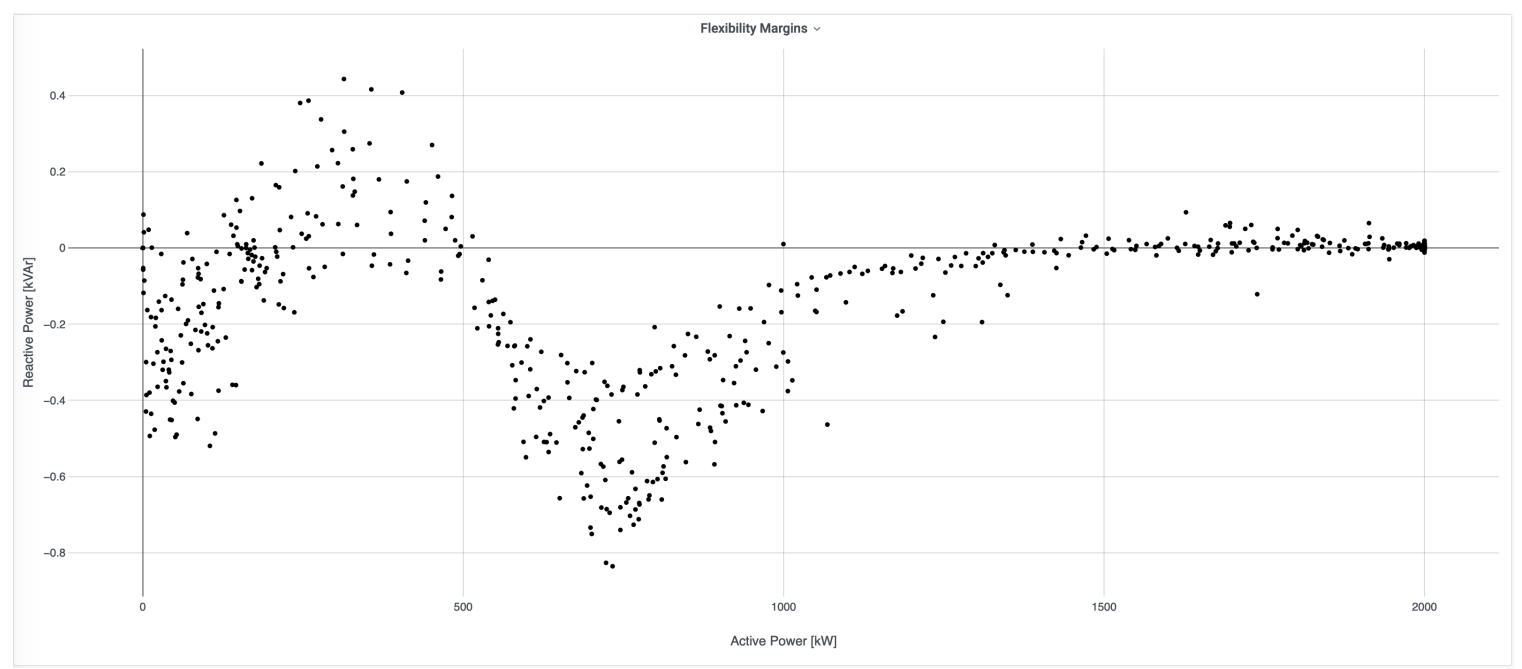

Fig. 7. Scatter plot showing real power vs. reactive power values over a range of operating conditions.

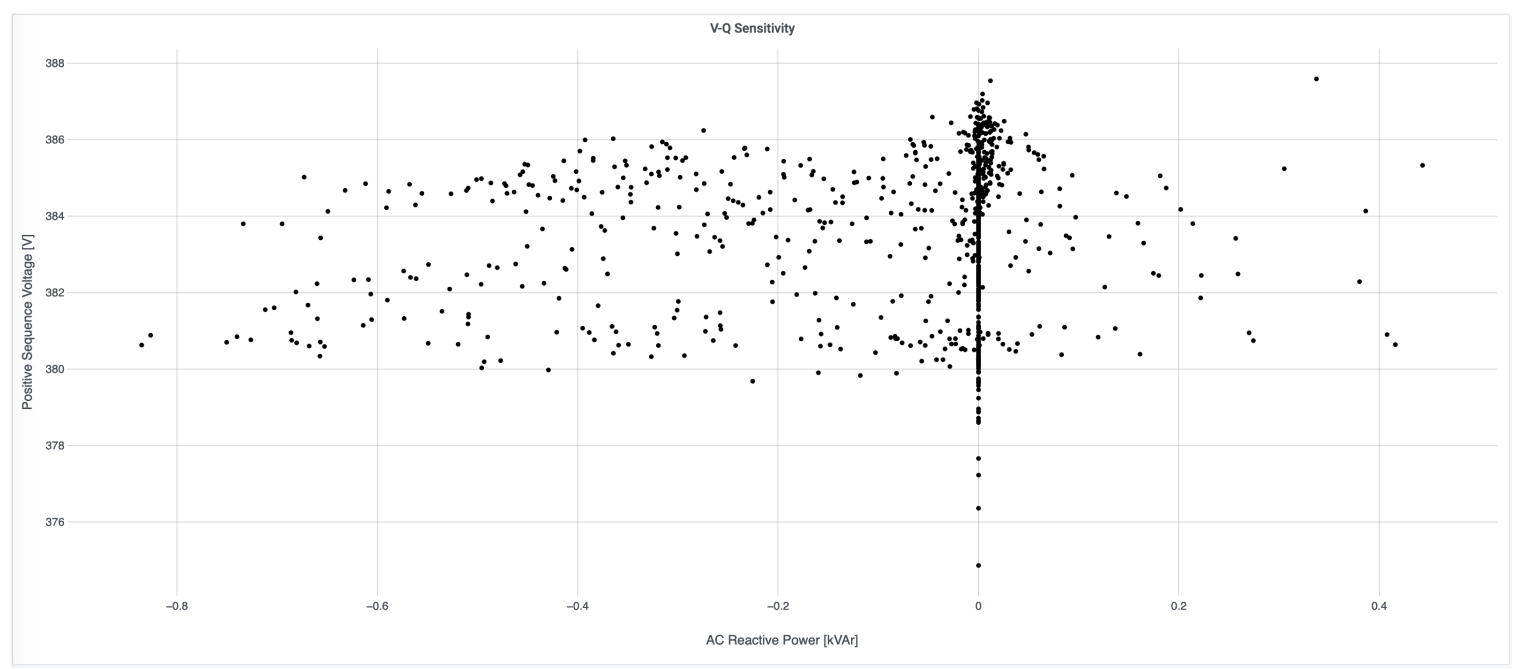

Fig. 8. Scatter plot showing voltage vs. reactive power values over a range of operating conditions. 


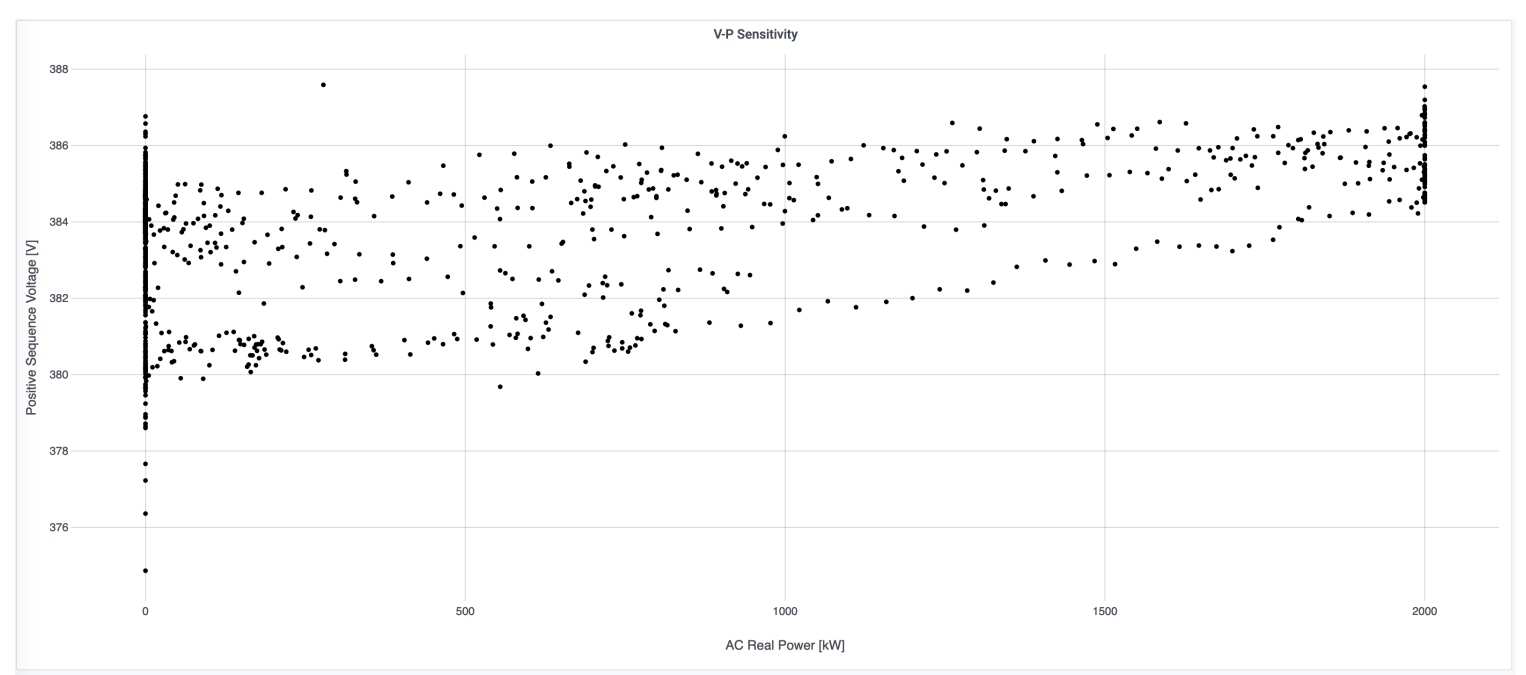

Fig. 9. Scatter plot showing voltage vs. active power values over a range of operating conditions.

Lastly, live monitoring of PV generators and the state of the campus grid as a whole requires continual queries of over 1000 data fields. Stress testing of the live monitoring feature was able to update the data dashboard in Figure 10 at a rate of twice per second.

\subsection{Communication Infrastructure}

The data pipeline described above primarily shares the campus area network with the existing computing and information technology infrastructure. Sensors and monitoring systems for building loads and switchgear are authorized, network connected hardware. All pipeline software components also meet security and software integrity standards to operate on the existing IT network.

In a few cases, specialized wireless communication links were installed for the purpose of communicating with DERs located outdoors. Here, care was taken to consider the wide range of wireless technologies used for communication and control of DERs in a distribution system. Examples from four commonly used classes of wireless communication were implemented on campus to transmit data from PV inverters, weather stations, substation relays and Phasor Measurement Units (PMUs).

Table 1 lists the salient features of each of the technology classes currently implemented. The first technology in the table is a Layer-2 Ethernet, Point-to-Point (P2P) system. This $5 \mathrm{GHz}$ system is intended to capture the bandwidth, security and reliability requirements of inter-substation, relaying and communication. The link is secured via a pre-shared key and operates between a PV generating site and a data server site.

The second technology in the table is a Wireless Local Area Network segment (WLAN). This system uses the IEEE 802.11ac wireless networking standard in the $2.4 \mathrm{GHz}$ band. This link was designed with enterprise security standards in mind and is primarily used to evaluate remote management and diagnosis functions. This system uses user authentication 


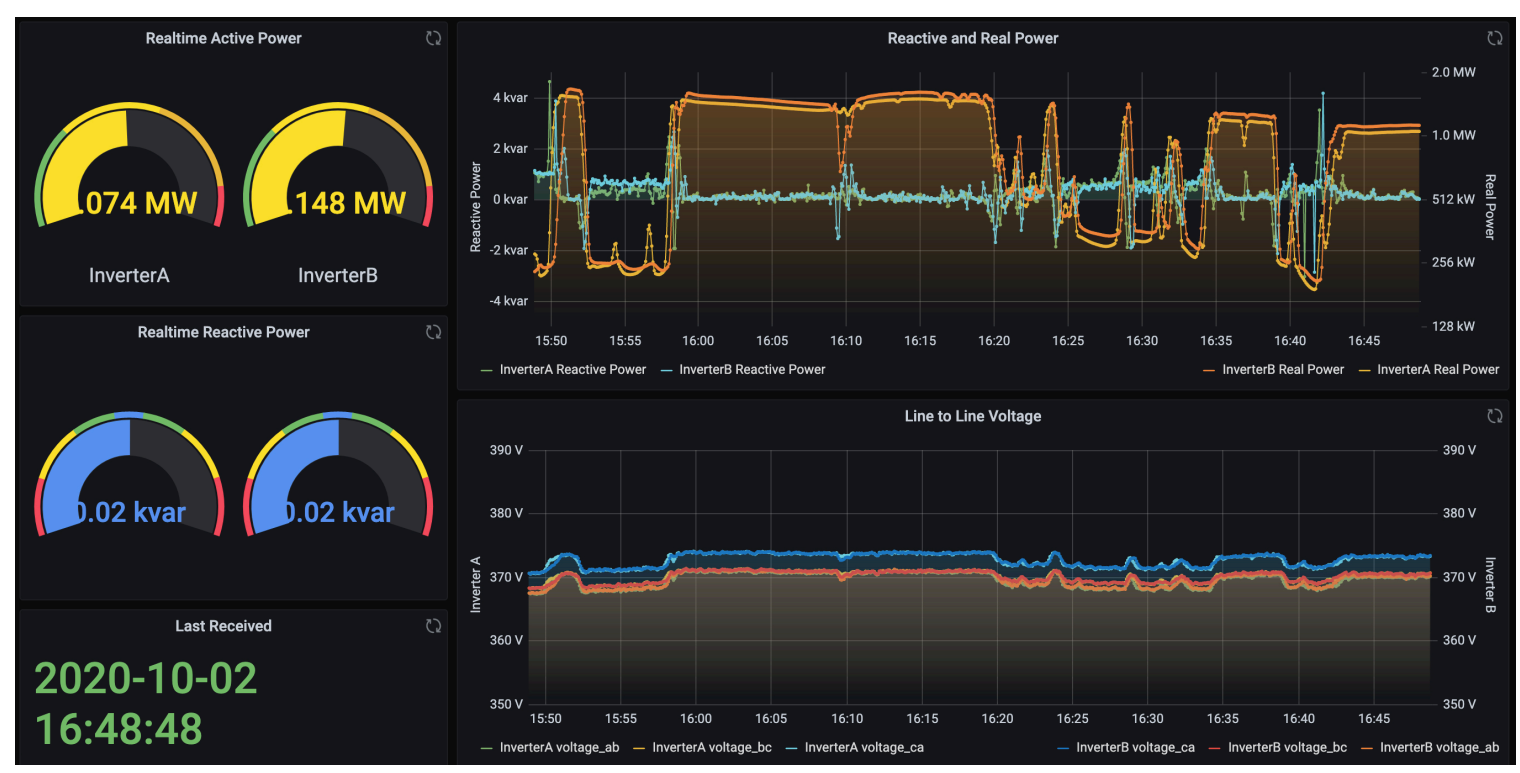

Fig. 10. A screenshot of a monitoring dashboard displaying the live behavior of one PV generator system.

and access policies, meeting the Extensible Authentication Protocol (EAP) requirements at NIST.

The third technology in the table is $900 \mathrm{MHz}$ system, employing a utility grade Supervisory Control and Data Acquisition (SCADA) functions in currently deployed Distribution Management Systems (DMS). This link has a significantly slower data rate but has historically been trusted for deterministic control transmissions to relays and tap changers.

The fourth technology listed in the table is a low-power wide area network (LPWAN). This link is significantly slower than all the other wireless technologies but provides over 10 kilometers of range with low power radios and other features that make it amenable to applications, requiring low power draw and low cost. LPWANs have been considered a feasible technology for residential-scale load monitoring, weather monitoring and asset tracking applications, where the need for ubiquitous sensing supersede the need for high speed communication.

The data pipeline can use any of the four wireless technologies listed, allowing experimenters to trade-off security overhead considerations with base data rate and latency requirements. For example, Figure 11 shows latency measurements taken from three of the high speed communication links available. Figure 11(a) shows the latency variation of the WLAN link. This link is observed to have periodic spikes in the latency, related to access and congestion constraints. Meanwhile, the P2P link shown in Figure 11(b) uses fixed, time division multiplexing and can ensure better determinism. However, it loses the resilience benefits of a networked wireless system, such as mesh routing or redundant access points. Figure 11(c) shows the latency variation of a wired $1000 \mathrm{Mb} / \mathrm{s}$ Ethernet link to a DER communication interface located in the field. This last plot provides a baseline for 
Table 1. Table showing salient features of the four classes of wireless technologies used on the NIST campus to support validation efforts.

\begin{tabular}{l|llll} 
Technology & P2P & WLAN & SCADA & LPWAN \\
\hline Approximate range & $5000 \mathrm{~m}$ & $100 \mathrm{~m}$ & $500 \mathrm{~m}$ & $10000 \mathrm{~m}$ \\
Usable datarate & $450 \mathrm{Mb} / \mathrm{s}$ & $450 \mathrm{Mb} / \mathrm{s}$ & $120 \mathrm{~Kb} / \mathrm{s}$ & $4 \mathrm{~Kb} / \mathrm{s}$ \\
Transmitter power & $25 \mathrm{dBm}$ & $20 \mathrm{dBm}$ & $16 \mathrm{dBm}$ & $20 \mathrm{dBm}$ \\
& $256 \mathrm{bit}$ PSK & $256 \mathrm{bit}$ EAP & $128 \mathrm{bit}$ & $128 \mathrm{bit}$ \\
Security standard & WPA2-AES & WPA2-AES & AES & AES \\
& $5 \mathrm{GHz}$ & $2.4 \mathrm{GHz}$ & $900 \mathrm{MHz}$ & $900 \mathrm{MHz}$
\end{tabular}

the performance of the pipeline.

\subsection{Grid-edge Computing Test Platform}

Another aspect of validating distributed systems is to consider the use of intelligence or autonomy at each DER site instead of a fully centralized system. The delegation of control functions to the edge of the grid, while ensuring reliable system level performance, is a research challenge that requires significant validation support. Assumptions about circuit models, local state estimates and computing capabilities must be exhaustively tested under all corner cases before grid operators will be willing to implement this fundamental shift in the control paradigm.

The architecture of the data pipeline in Figure 5, shows a clear demarcation of the logical boundary in the design. The pipeline components are distributed between "Field Servers" and "Data Servers." Field Severs in the diagram refer to computing resources physically co-located with DERs in the field. A custom designed computing test platform was designed and implemented as part of the campus validation effort. Figure 12 provides a schematic overview of the developed platform. This design is used for all the Field Servers currently deployed on campus.

The hardware used is a general purpose, fan-less computer (Model TWB-2945), built on an Intel N2930 1.8 GHz processor. This computer is housed in a ruggedized, weather resistant (IP-67 rated) chassis. The computer features two WLAN interfaces, two Ethernet interfaces and three serial interfaces, in order to communicate with components of the data pipeline as well as the wide variety of physical interfaces used for DERs. Ruggedized and weather-rated M12 connectors are used for electrical supply and data connections.

Of particular note, the presence of both serial and Ethernet interfaces allow simultaneous implementation of the two major variants of the Modbus protocol. Modbus TCP transfers data points over Ethernet and Modbus RTU typically uses RS-485 serial signaling. This capability allows one grid-edge computer to simultaneously interact with a PV inverter, using Modbus TCP, and PV module sensors, using Modbus RTU.

The operating system on the grid-edge test platform is Linux (Ubuntu Server 20.04). To facilitate easy reconfiguration of drivers communicating with the range of available hard- 

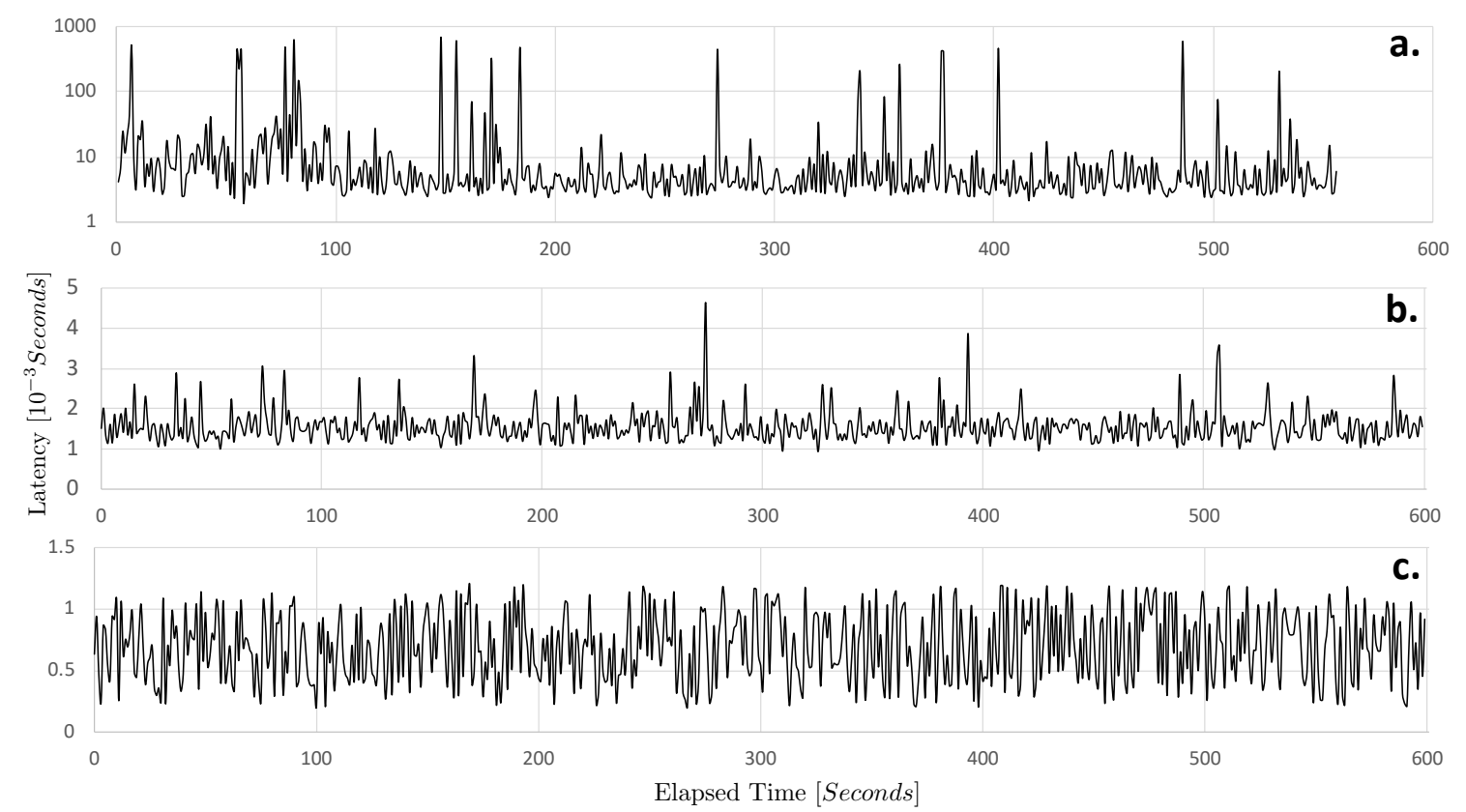

Fig. 11. A comparison of latency measurements for three high speed communication links. Subfigures a, b and c correspond to WLAN, P2P and wired Ethernet links respectively.

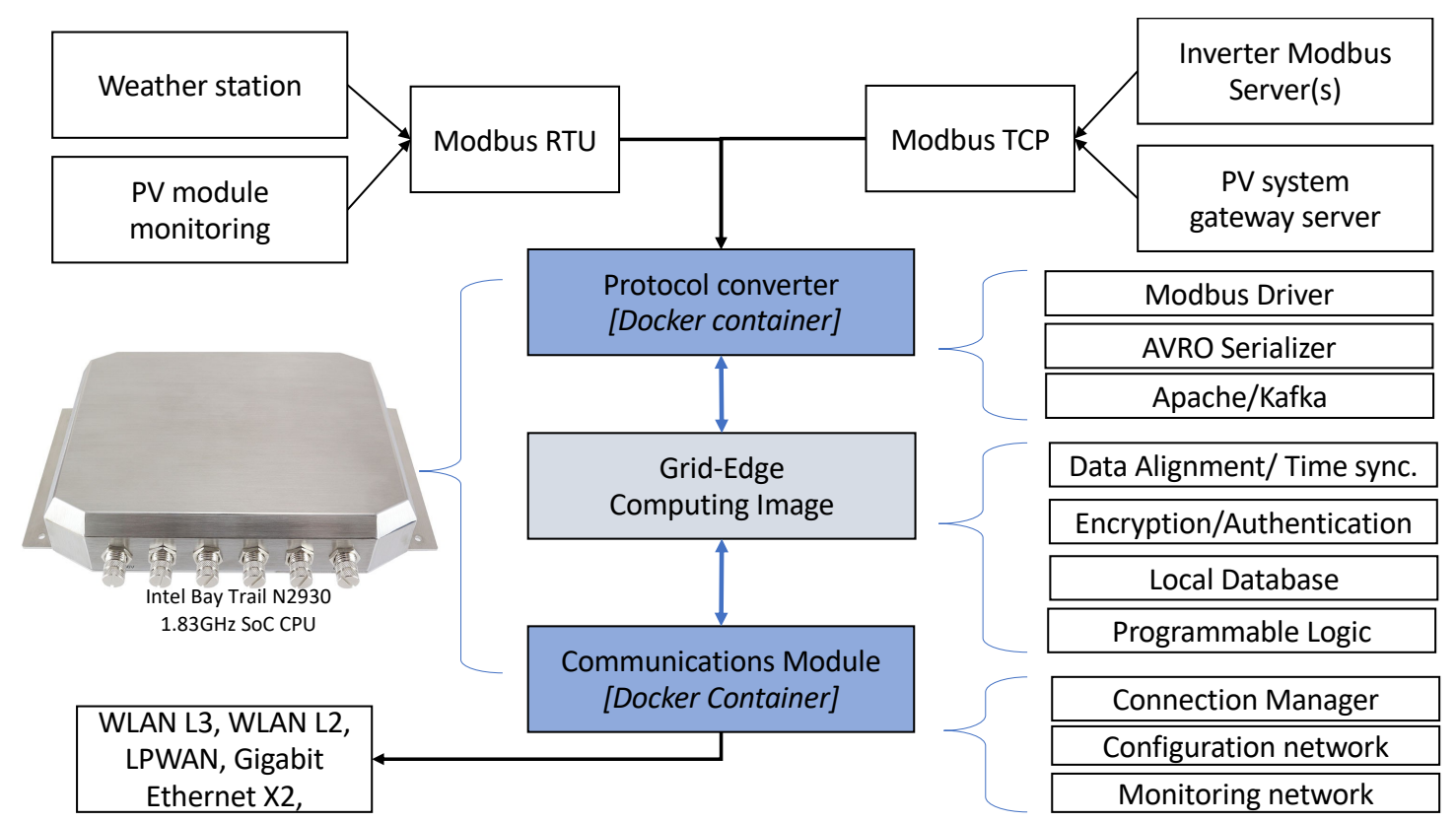

Fig. 12. A schematic diagram showing the key components of the NIST developed test platform for grid-edge intelligence. Image of the TWB-2945 fanless computer is used with permission. 


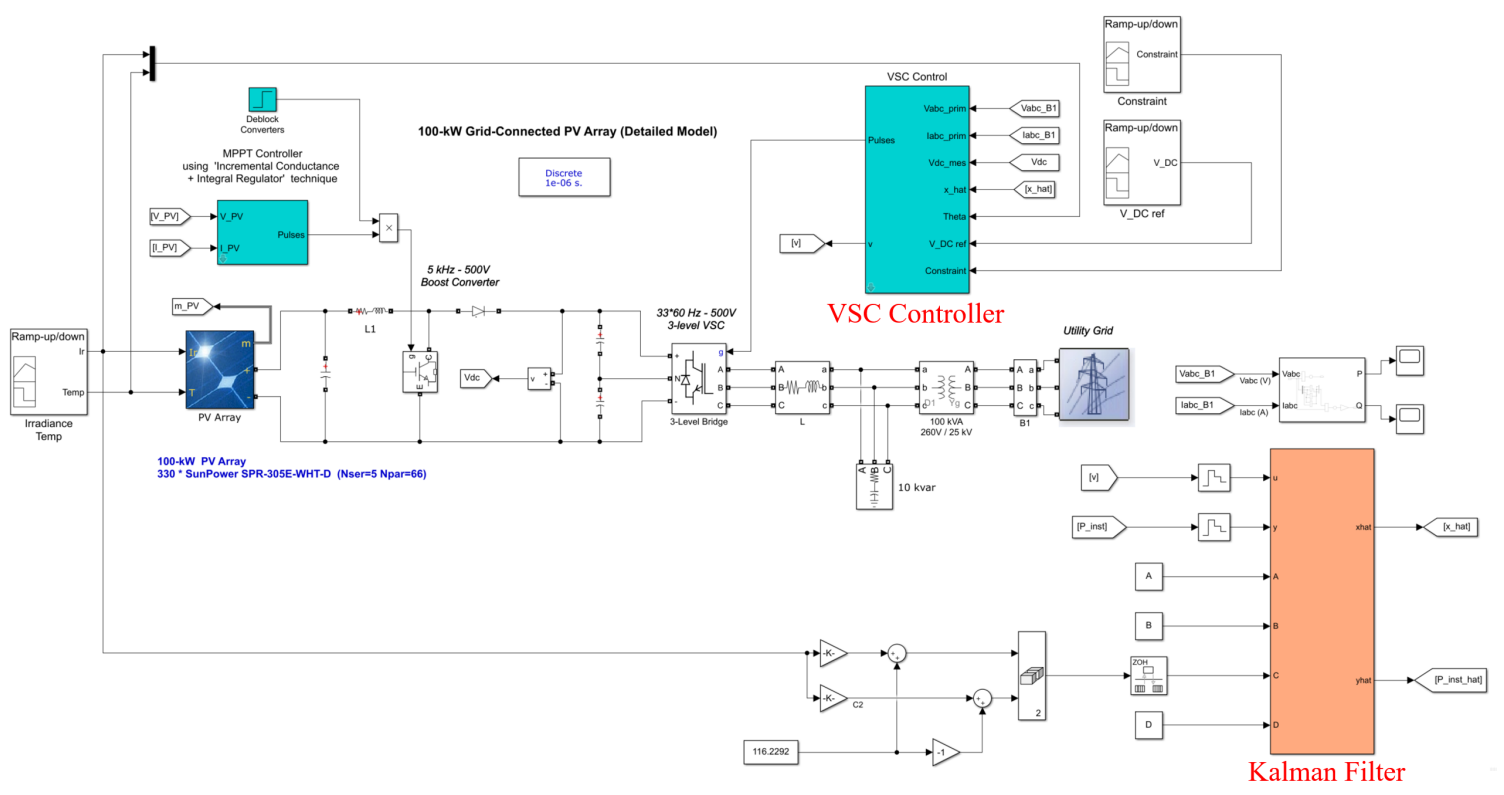

Fig. 13. RG-AC governed Detailed Model of a $100 \mathrm{~kW}$ Grid-Connected PV Array in Simulink. Some of the blocks shown in this diagram are derived from a model in Mathworks' Simscape Electrical library.

ware interfaces, the operating systems host a Docker virtualization engine [79]. All of the drivers used for communication are packaged together as a Docker container application, allowing easy replication and deployment.

This virtualization approach also greatly simplifies the implementation of the protocols and software parsers needed to communicate with commercial DERs and converts them to protocols used by distribution system operators (such as IEC 61850). Experimenters can develop a Docker container application with all of the protocol conversion software needed and then transfer the application as a single package to a field server.

The operating system on the field server provides a general purpose programming environment to test-distributed control algorithms using real data from a connected DER. As a demonstration of this capability, the Adaptive Reference Governor described in Section 4.2 was tested against a grid-tied PV inverter ${ }^{7}$. The algorithm was developed on the Matlab/Simulink platform in the laboratory and compiled into software binary that could be deployed to a field server. 


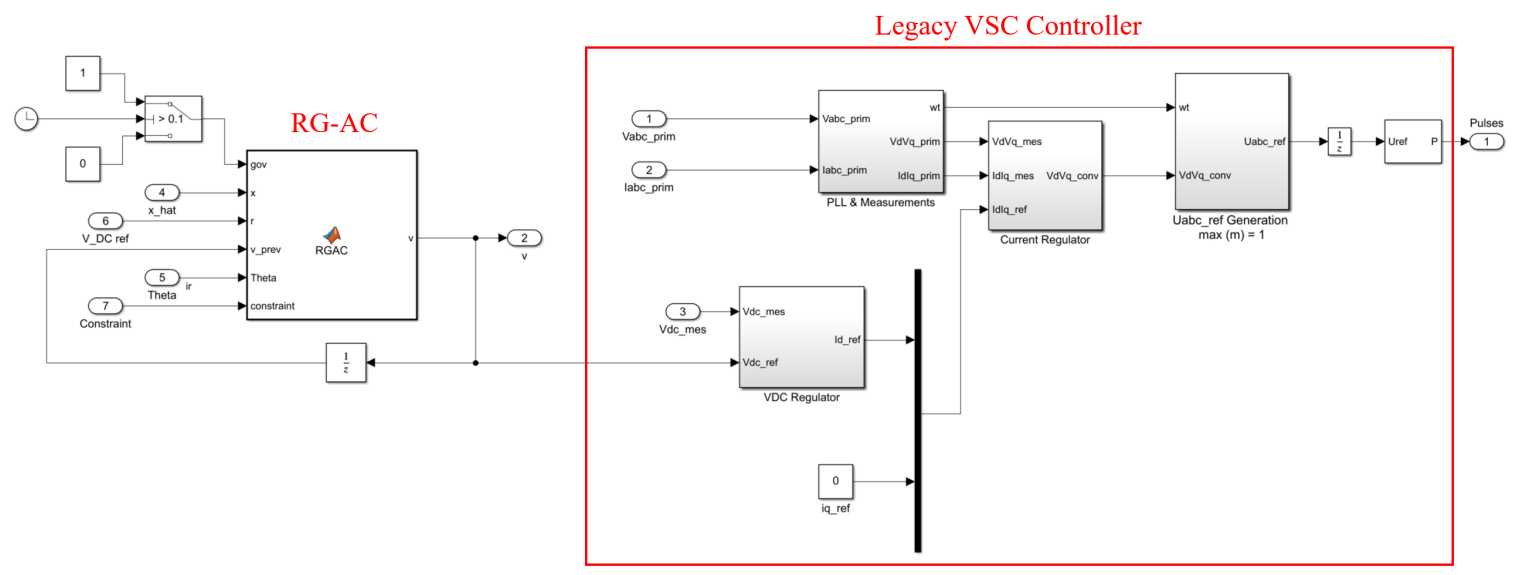

Fig. 14. Detailed view of the VSC Controller block from Figure 13 showing the addition of the Reference Governor.

\subsubsection{Adaptive Reference Governor for Grid-tied Photovoltaic Inverters}

The Adaptive-Contractive Reference Governor (RG-AC) was applied to a "Detailed Model of a $100 \mathrm{~kW}$ Grid-Connected PV Array" [80] implemented in Simulink ${ }^{8}$. Screenshots of the Simulink model used for simulation are shown in Figs. 13 and 14. The control objective in this evaluation was to govern the real power exchanged with the grid to demonstrate a constraint aware implementation of the Volt-Watt function described in Section 4.1.

$\mathrm{RG}-\mathrm{AC}$ requires a discrete-time state space model of the ungoverned, closed-loop system to enforce constraints. Thus, a model of the PV inverter was developed using a "black-box" approach to estimate the inverter dynamics from the DC bus voltage reference $\left(V_{D C, r e f}\right)$ to the instantaneous real power $(P)$ delivered to the grid. Solar irradiance greatly affects the dynamics of PV inverters. Specifically, it affects the amount of power that a PV system can produce. Thus, to effectively enforce constraints using RG-AC, the system model must account for the change in the inverter dynamics as a function of the solar irradiance. To achieve this, linear models were developed for various constant values of solar irradiance, allowing for realization of the effect of solar irradiance on the model parameters. In final, a linear, parameter-varying model was generated, with the time-varying parameter being the solar irradiance, measured in $\frac{\mathrm{W}}{\mathrm{m}^{2}}$. The system model, with a sampletime of 0.1 milliseconds, is shown in Eq. (1) below:

$$
\begin{aligned}
x(t+1) & =A x(t)+B u(t) \\
y(t) & =C x(t)+D u(t)
\end{aligned}
$$

\footnotetext{
${ }^{7}$ While measurement data and configuration settings of one of the PV generators on campus were used for this validation study, control scenarios were implemented on a validated model of an inverter in compliance with physical access and safety policies.

${ }^{8}$ This model is available to download as part of a commercial software library from Mathworks. https://www.mathworks.com/help/physmod/sps/ug/detailed-model-of-a-100-kw-grid-connected-pvarray.html;jsessionid=1032e3b4c9b3dc9462faf215a2ce\#responsive_offcanvas
} 

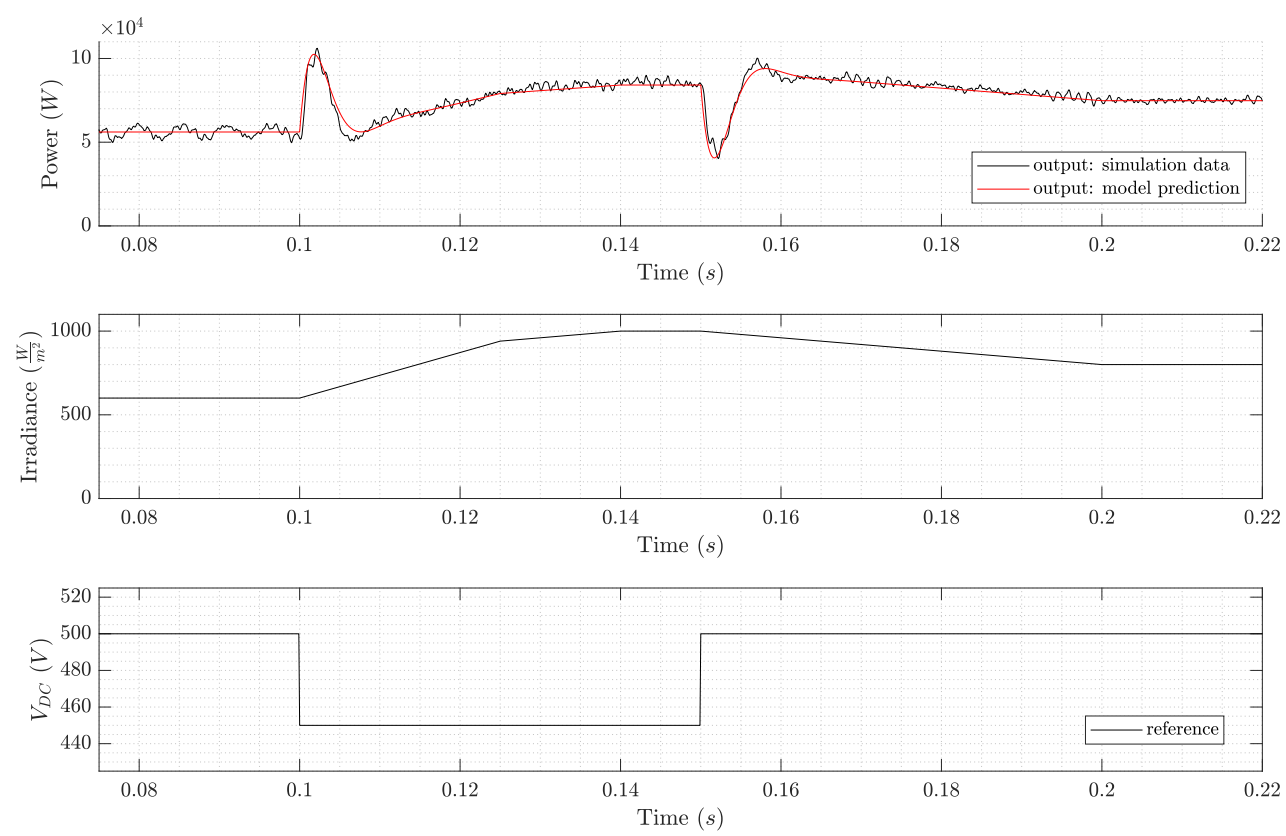

Fig. 15. Linear parameter-varying PV inverter model compared to simulated output. Positive power corresponds to power being delivered to the grid.

where

$$
\begin{aligned}
& A=\left[\begin{array}{cc}
0 & 1 \\
-0.9220 & -1.9182
\end{array}\right], \quad B=\left[\begin{array}{l}
0 \\
1
\end{array}\right], \\
& C=\left[\frac{0.3459}{1000} \Theta(t)+116.2292 \quad \frac{0.3554}{1000} \Theta(t)-116.2292\right], \quad D=0
\end{aligned}
$$

and $\Theta(t)$ is the sun irradiance in $\frac{W}{m^{2}}$. A comparison of the model's output and the simulated system's output is presented in Figure 15. Note that the predicted power given by the model matches the simulated system's power closely, despite the time-varying nature of solar irradiance.

Figure 16 compares the responses of the governed and ungoverned PV inverter systems subject to the same initial conditions, reference signal, solar irradiance, and constraint. The constraint in this example is applied to the inverter output power for the purpose of limiting the real power exchanged with the grid. At the beginning of the simulation, the constraint is $P<100.0 \mathrm{~kW}$, and immediately ramps down to $P<90.5 \mathrm{~kW}$ by the 0.35 second mark. Here, it remains constant for the rest of the simulation. Furthermore, the solar irradiance rapidly fluctuates between $600 \frac{\mathrm{W}}{\mathrm{m}^{2}}$ and $1000 \frac{\mathrm{W}}{\mathrm{m}^{2}}$ throughout the simulation.

Despite the exaggerated time-varying nature of the inverter dynamics and the constraint, the RG-AC governed PV inverter does not violate the established constraint, whereas the ungoverned inverter violates the constraint multiple times. These results are promising because it suggests that an external controller can supply individual inverters with constraints 

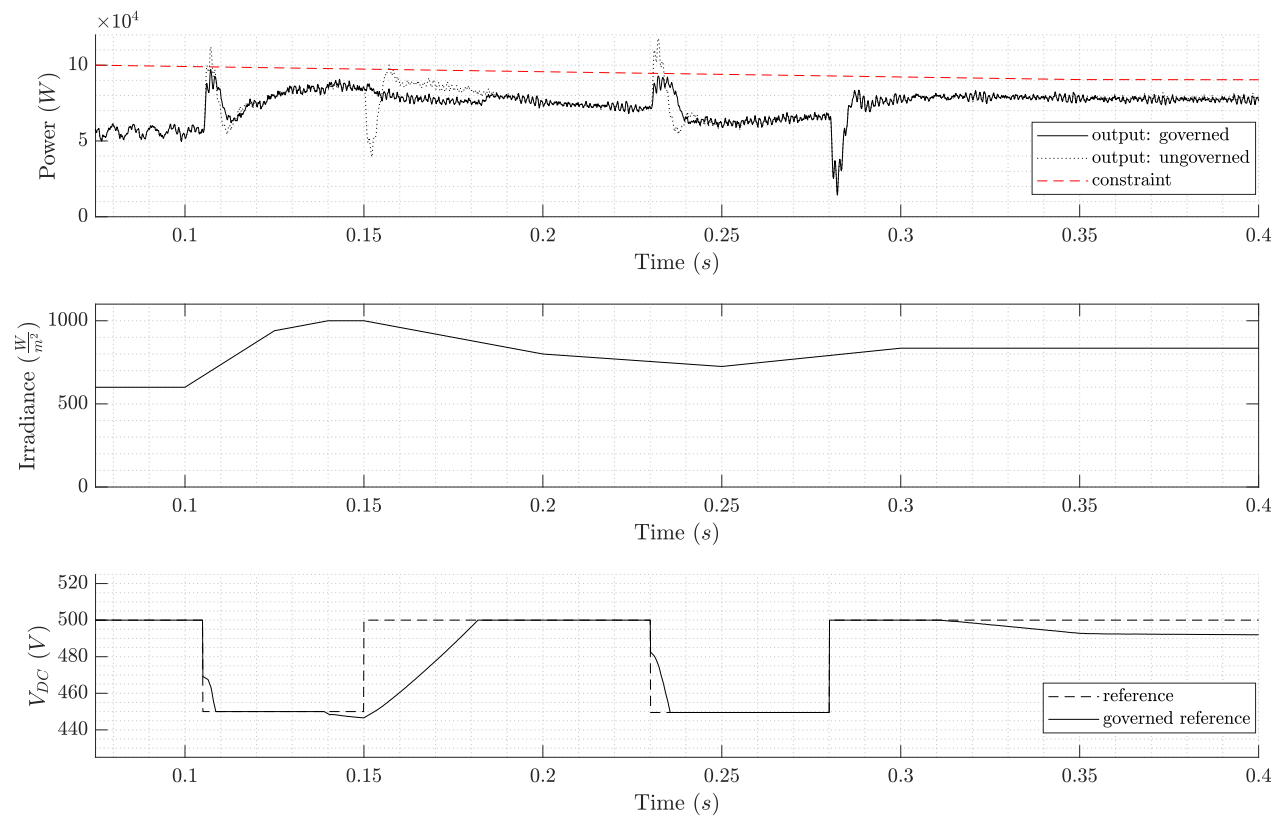

Fig. 16. RG-AC governed PV inverter test results. Note that the reference bus voltage $V_{D C}$ is governed resulting in a successfully constrained response of the power output.

and references, knowing that each inverter will track the references as closely as possible without violating the constraints. This study demonstrates that the distributed controller developed in Section 4.2 is successful on a "detailed," validated simulation grade model of an inverter system. An important milestone in developing an engineered solution for constraint enforcement is achieved that enables composition of distributed assets as envisioned in Section 1.

The analysis capabilities of the grid-edge computing test platform presented in Section 3.4 allows extension of the analysis to aspects of performance that may not be initially considered. In the case of the RG-AC PV inverter controller described above, the simulation model assumes a balanced three-phase system. Simulation studies show that constraint enforcement is successful under this assumption. To test this assumption, an analysis module was installed on the grid-edge computing test platform to measure the voltage symmetry of the point of common coupling of the PV inverter system as installed. Figure 17 shows a scatter plot of line to line voltages observed at the point of coupling at one of the campus PV generator sites. The figure shows a consistent, though small, offset between $V_{C A}$ and $V_{A B}$ when compared to $V_{B C}$, whereas the simulation study does not consider this offset as shown in Figure 18.

This is an expected deviation between the simulated DER system and the real DER system since the simulation was only validated against an inverter under symmetric loading conditions. The impedance of the point of coupling in the PV generator on campus is not 
identical across all three phases.

The grid-edge test platform enabled this analysis to be conducted directly at the DER site eliminating the need for reconfiguration or modification to the data pipeline. The measurement of imbalance thus obtained can be used to update the constraints for the RG-AC controller.
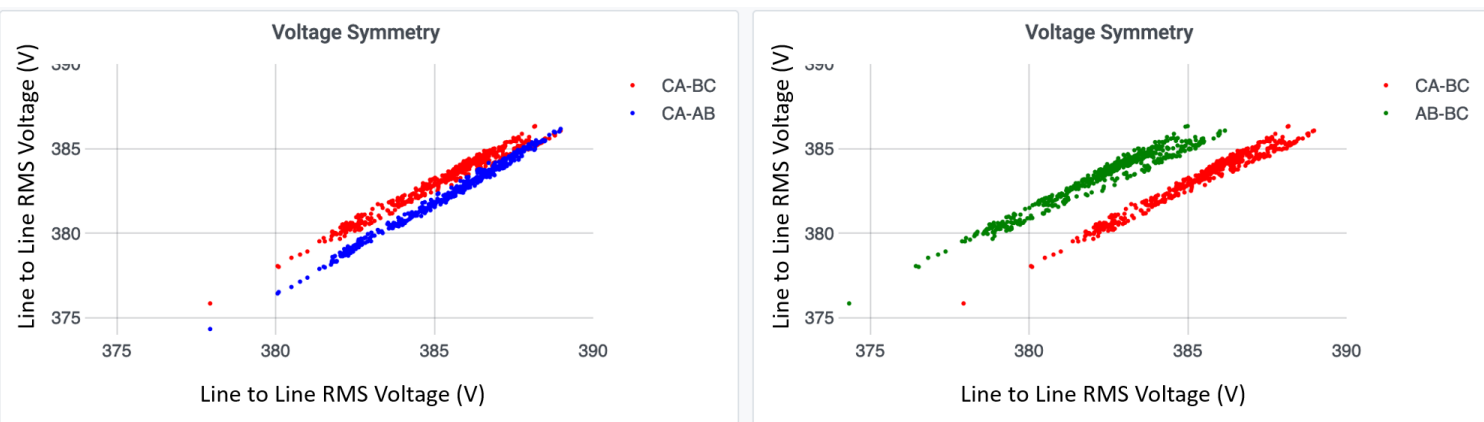

Fig. 17. A scatter plot showing line to line voltage variation observed at one of the campus PV generator sites measured over the full Volt-Watt control range. Two sets of scattered points shown on each plot correspond to $V_{C A}$ versus $V_{B C}$, and $V_{C A}$ versus $V_{A B}$ respectively.
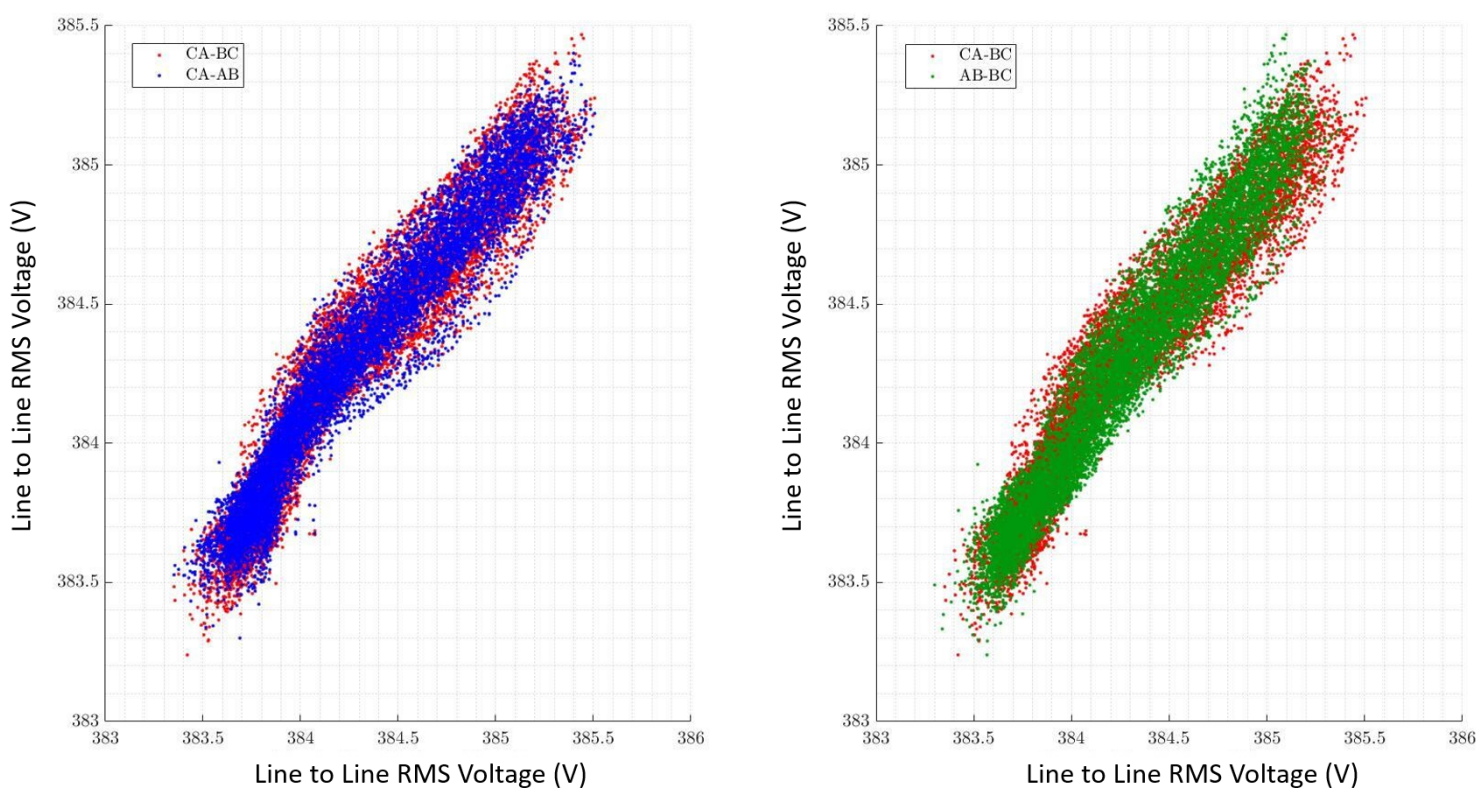

Fig. 18. A scatter plot showing line to line voltage variation assumed in a simulated PV generator over the full Volt-Watt control range. Two sets of scattered points shown on each plot correspond to $V_{C A}$ versus $V_{B C}$, and $V_{C A}$ versus $V_{A B}$ respectively. 


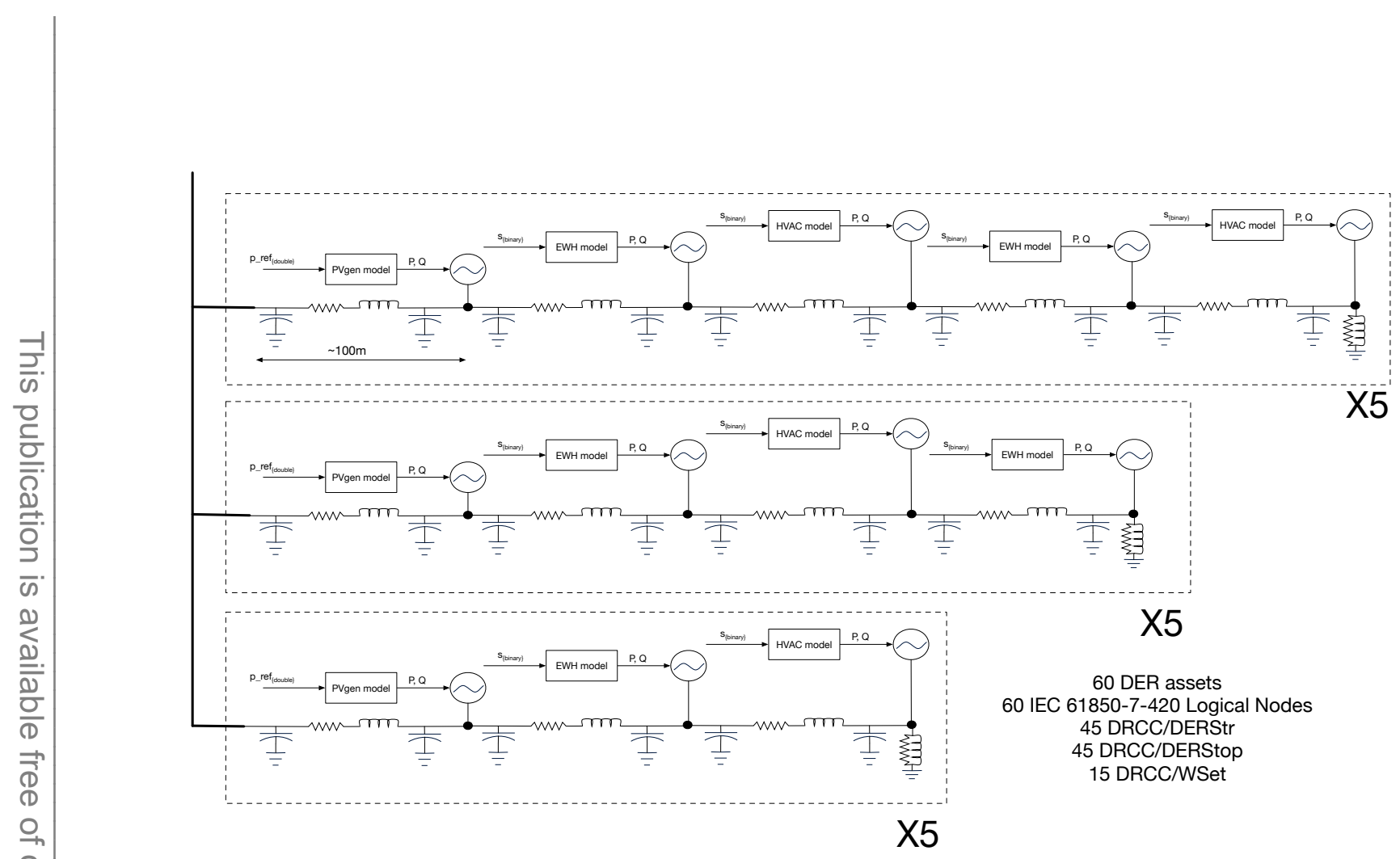

Fig. 19. DER models with corresponding DER Unit controllers simulated in real-time using $1 \mathrm{~ms}$ timesteps and 10ms control loops.

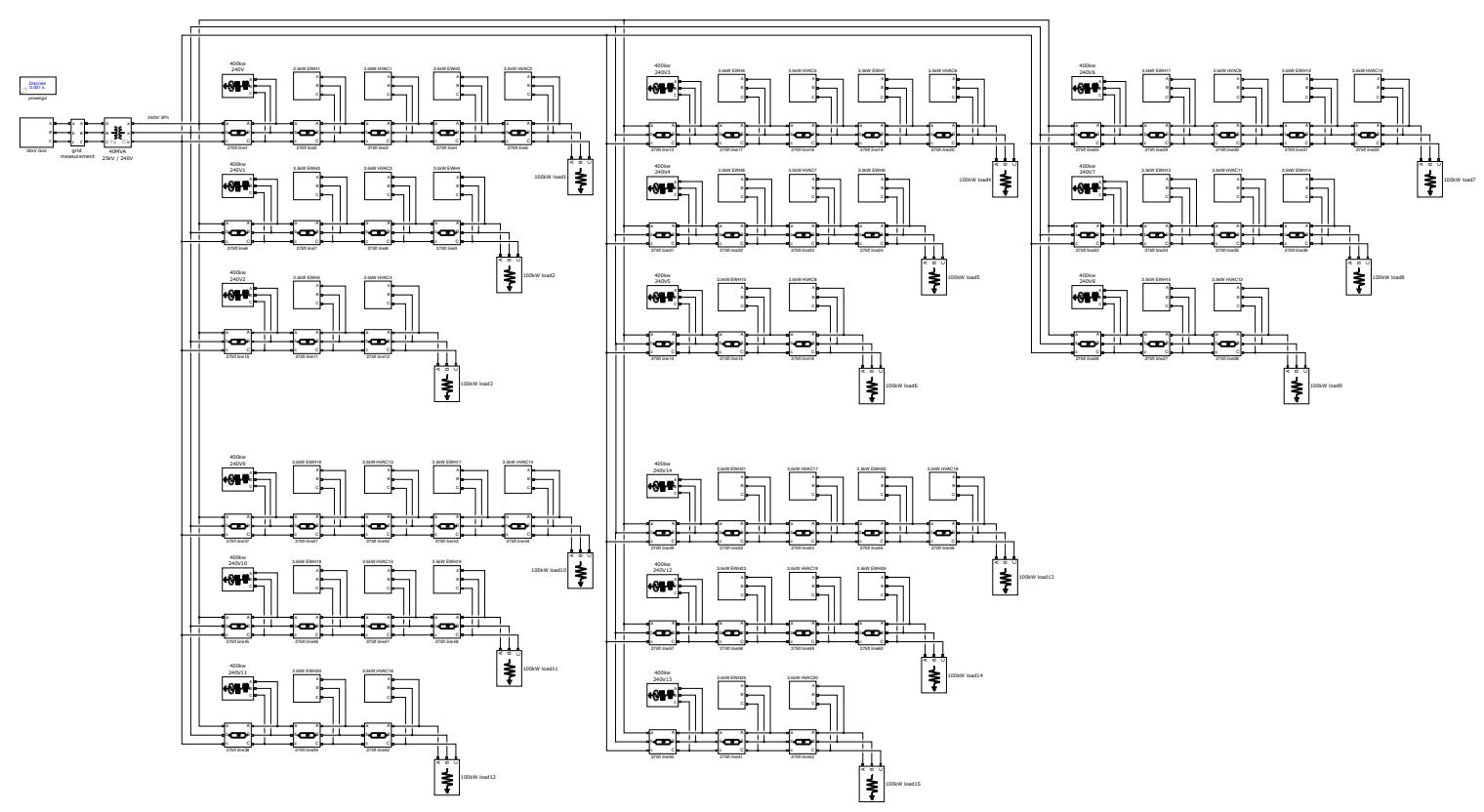

Fig. 20. Simscape Electrical model of 60 DERs forming proposed experimental architecture as per the schematic described in Figure 19. 


\subsection{Campus in the Loop Experiments}

The primary goal of the NIST campus validation infrastructure is to emulate operating conditions, test cases and datasets to support all of the research directions discussed in Section 4. The objective is to accomplish this goal using the infrastructure's subsets of measurements, the data pipeline infrastructure and the variety of computing resources, all together.

However, there are some experiments where the data pipeline infrastructure and DER interfaces themselves can serve as validation artifacts for real communication latency, delay and bandwidth constraints. The campus data pipeline infrastructure can operate hardware' in a hardware-in-the-loop experiment with simulated power system models and control algorithms. This allows experiments to evaluate scalability concerns of their algorithms and models.

This method of evaluation was used to test a coordinated Volt-Watt controller for 60 DER assets in a simulated distribution system. Particular emphasis was placed on capturing realistic latency and scale and time synchronization implications of interactions between communication networks, data exchange formats, information models and the fast time scale dynamics of controllers at the feeder and transformer levels. Figure 19 shows the overall scale of the experiment. A single distribution circuit model was constructed containing 60 DER assets, connected to 15 radial lines, bearing a service transformer on each, supplied from a single head end substation. The DER assets modeled were a mix of PV generators and dispatchable loads in the form of electric water heaters and HVAC units.

Each DER asset was also modeled as an IEC 61850-7-420 logical node [81, 82], receiving individual power set points and configuration settings from the DER coordination system. These measurements and commands were modeled as IEC 61850-7-2 data objects, exchanged as IEC 61850-8-1 Generic object-oriented substation events (GOOSE) [83]. The full model, including dynamic representations of the DER assets and the IEC 61850 components, was modeled using the Simscape Electrical library. This model was optimized for real-time simulation and connected to software drivers for IEC 61850-8-1 communication. The full model was run on an Opal-RT OP5600 real-time simulator with deterministic loop execution time of $10 \mathrm{~ms}$. A screen shot of the full model is shown in Figure 20.

An overview of the communication requirements of this simulation is shown in Figure 21. Communication of set points and DER state, to and from each DER, to the disaggregation component of coordination controller, requires 1800 messages per minute. The disaggregation controller then updates transformer load state, using IEC 61850-8-1 Manufacturing Message Specification (MMS) messages, and receives set points from the distribution management system at a rate of 120 messages per minute. The temporal scaling between the communication and computation elements ranges from a $10 \mathrm{~ms}$ loop time for individual DERs, 4 second computations at the service transformer level (STL), leading to minute scale optimization at head end feeder operational layer (FOL). All these computational updates are locked to the same hardware clock, ensuring they are coherent with each other. 


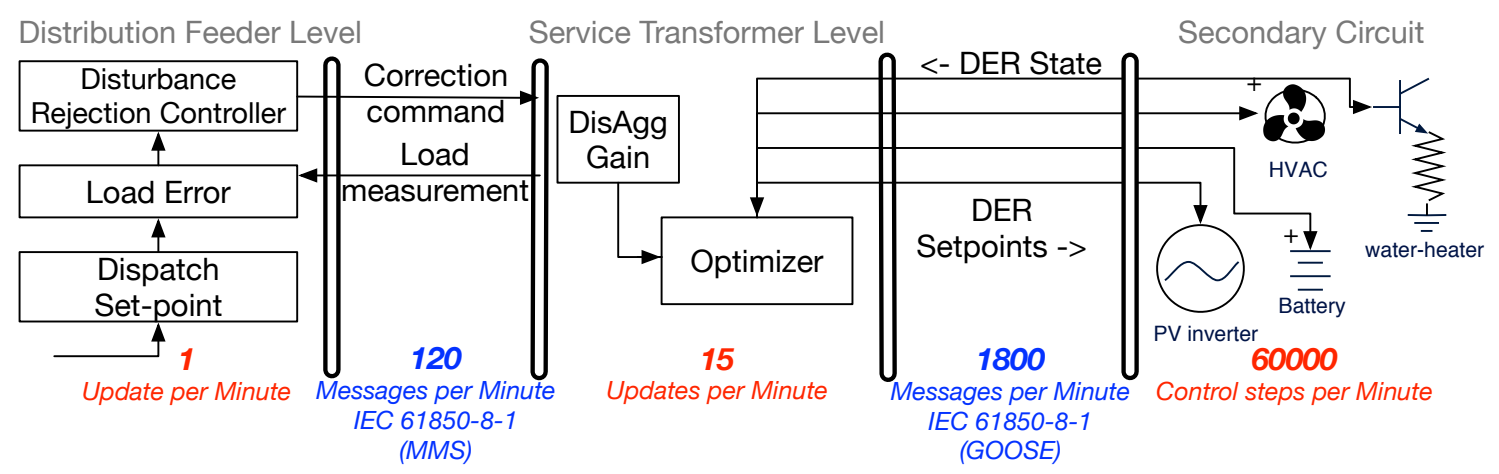

Fig. 21. A schematic diagram showing the interacting elements in the validation environment. Here a single feeder and service transformer unit are considered. 60 DER assets are coordinated by the system using IEC 61850 compliant information models and communication protocols.

The validation aspect of this exercise was establishing whether potential congestion from rapid, periodic updates would adversely affect the performance of the control system at the FOL and STL. Here, real-simulation of the power system and the controller was implemented as a client application (see Figure 5). Outgoing IEC 61850 messages produced by the simulation were routed through the data pipeline as Kafka topics and were eventually recorded in the Postgres database. Stream processing on the database then generated a query to push the data back through the data pipeline to eventually arrive as an incoming IEC 61850 update to the simulation.

Since the data pipeline was designed to accommodate a full scale distribution system, the messages routed through the system acquire delays and errors that a real system would encounter. This provided valuable insight on the scalability of the control scheme being tested in simulation.

Figure 22 shows the throughput of the data pipeline while performing this experiment. Figure 22(a) shows a histogram of packet arrival rate. The x-axis on the plot shows the total time taken for all 60 DERs to transmit their state and receive commands from the STL. In this particular instance, all 60 DERs were updated in under the update loop time for the STL of 4 seconds. The average latency of the updates was $5.2 \mathrm{~ms}$ which is below the update time of $10 \mathrm{~ms}$ for each DER. Similarly, Figure 22(b) and Figure 22(c) show the stable throughput exceeding the update constraints of the FOL with bounded message latencies of approximately $10 \mathrm{~ms}$. 

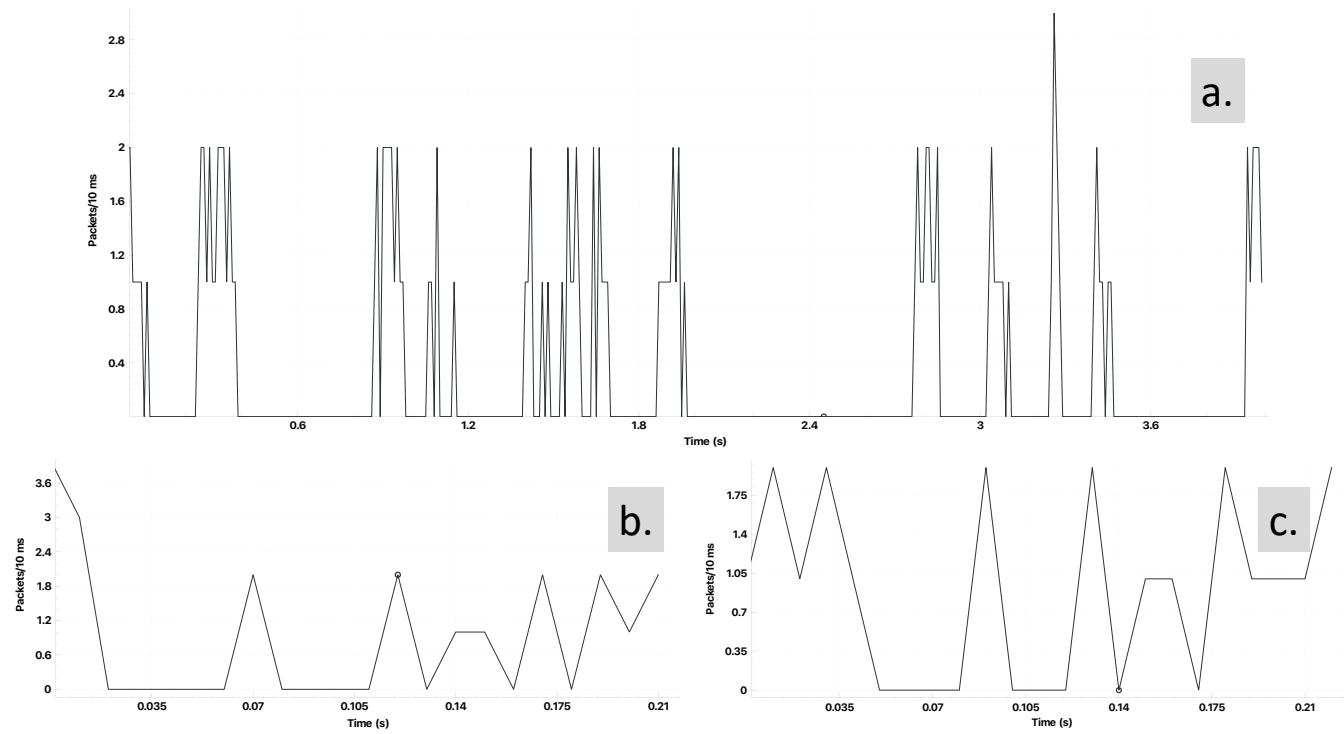

Fig. 22. Communication throughput: a. GOOSE exchanges between STL and DERs (average latency $\sim 5 \mathrm{~ms}$ ) b. Load request correction from FOL to STL (average latency $\sim 10 \mathrm{~ms}$ ) c. Load estimate query from STL to FOL.

The data pipeline appeared to adequately meet the update rates required for the control system used in this experiment. The performance of load tracking functions of the system are shown in Figures 23 and 24. Figure 23 shows the reference set point, provided by the FOL, the control signals generated by the STL and the successful response of the full fleet of 60 DERs in tracking the desired set point.

Figure 24 magnifies the plot in Figure 23, showing the transient response of the fleet of DERs, in response to load change requests every 4 seconds. The figure illustrates momentary transients in the load response, which are neither sustained or unstable, suggesting this system would be stable in real world operation.

The validation experiment discussed in this sub-section considers the stability of the control system, the stability of the dynamic response of DERs, as well as the stability of the communication infrastructure. This is an example of the type of system level validation that considers both cyber and physical components and addresses the concerns raised in Section 1.

\section{Areas of Research and Development for Validation}

This section outlines research projects currently underway within the NIST Smart Grid Program that leverage the campus instrumentation capabilities described in Section 3 to address the three major needs identified in Section 2. 


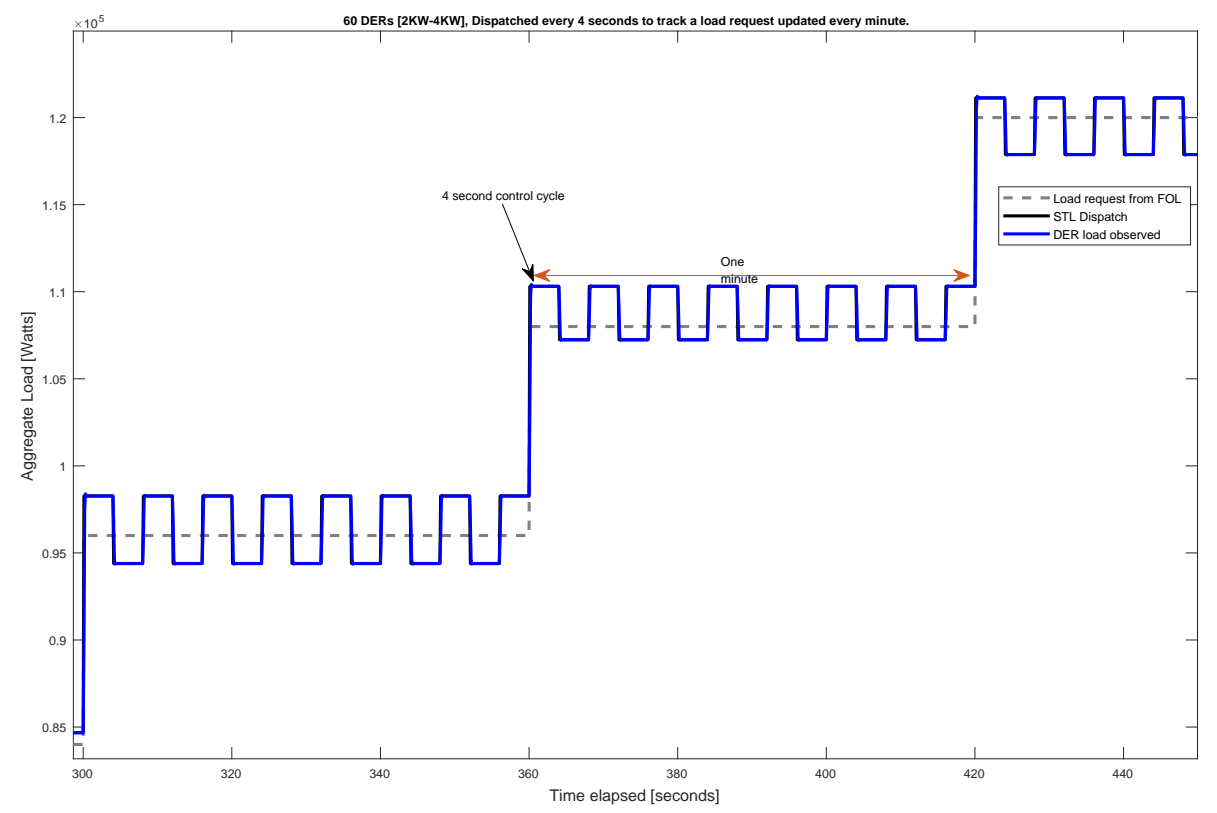

Fig. 23. DER dispatch performance showing the FOL set point and the corresponding updates generated by the STL. The cumulative load of all 60 DERs is also shown. The figure shows that the mean value of DER loads averaged over the one minute FOL update window tracks the requested set point successfully.

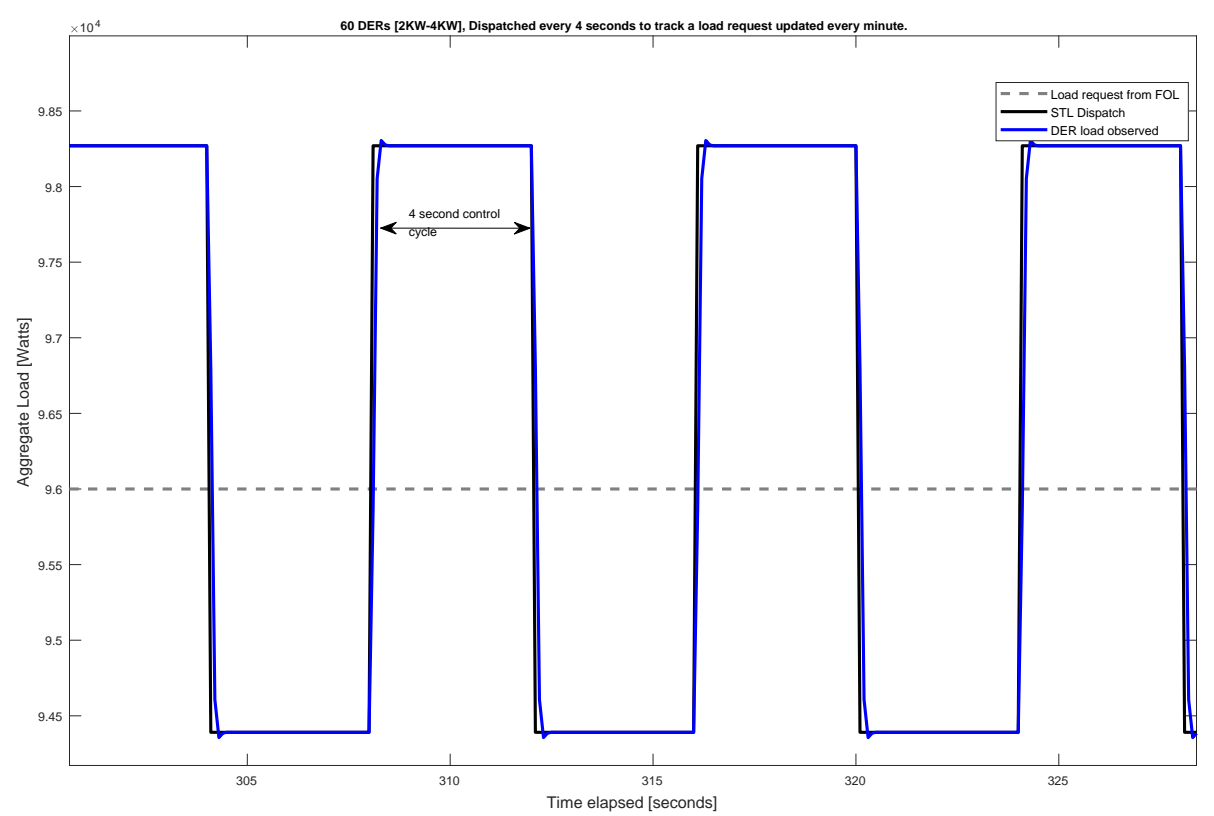

Fig. 24. A closer view showing the DER response to set point changes from the STL. The figure shows minimal overshoot in response to the $0.25 \mathrm{~Hz}$ update cycle. Note that the sustained $0.25 \mathrm{~Hz}$ tracking oscillation was added to the control system in order to investigate potential adverse interactions between the control system and the communication system. 


\subsection{Information Model Harmonization}

This NIST project seeks to harmonize information models in order to help DSOs integrate groups of DERs on their primary and secondary feeders into an existing Distribution Management System (DMS) in a scalable manner while still retaining analytical capabilities for testing, uncertainty analysis and control.

This work decomposes system-level functions of protection, automation and control into functions that can be performed by distributed assets. At the same time, this work seeks to adequately represent the capabilities and properties of individual DER assets in a manner that supports assembly or composition. A series of recent standardization efforts have improved the interconnection and interoperability between utility DMS and DERs. The IEEE 1547 standard [70] and its recent revision in 2018 [84] provide requirements relevant to the performance, operation, testing, safety, and maintenance of the interconnection.

Decomposing the functions specified in this standard can be challenging. DER assets may have varying communication requirements, different physical constraints, and may require information from adjacent DERs as well as the DMS. The functional decomposition will also likely include existing or legacy substation components and distribution circuit infrastructure components.

Electrical substations with digital controls and communication capabilities are commonly expressed using the information models in the International Electrotechnical Commission (IEC) 61850 group of standards. The generic object-oriented building block for representing function elements, per the standard, is called a "logical node". The logical nodes are grouped in hierarchical, logical devices which may have multi-level hierarchy, depending on the complexity of the functionality of the DER.

Recently, IEC Technical Committee 57 Working Group 17 has been working to develop IEC 61850-7-420. This extends the generic logical node model to DERs. Working Group 17 has given attention to the variety of communication services needed to support both client-server and peer-to-peer communications in order to meet the requirements of traditional energy management functions as well as the high-speed performance for protection applications.

The two standards described above represent a common theme in the development of the next generation of DER-based distribution systems. Simultaneous innovation on the electrical engineering and the information technology components must be supported while ensuring harmonization of terminology, functions and models.

The IEEE 1547 series of standards describes over a hundred specific tests, functions, fault behaviors and capabilities for DER and DMS systems. An illustrative example is Voltage - Active Power Capability, where a DER is required by IEEE 1547 to actively limit its active power output, as a function of the voltage, while following a Volt-Watt, piecewise linear characteristic. Two classes of Volt-Watt characteristic curves specified in the standard are illustrated in Figure 25.

This piecewise, linear function is modeled in the standard, using the six parameters and their corresponding limits shown in Table 2.

The standard also lists the four classes of mandatory information that each DER asset 
Table 2. Configurable parameters for the Volt-Watt function as specified in the IEEE 1547 Standard. Per unit voltage values are normalized to nominal grid voltage $V_{\text {nominal }}$.

\begin{tabular}{|l|c|c|}
\hline \multicolumn{1}{|c|}{ Parameter } & $\begin{array}{c}\text { Default value } \\
\text { (normalized to } \\
\text { per } \text { unit values) }\end{array}$ & $\begin{array}{c}\text { Permitted values } \\
\text { (normalized to } \\
\text { per } \text { unit } \text { values) }\end{array}$ \\
\hline$V_{1}[$ Volts] & 1.06 & 1.05 to 1.09 \\
\hline$P_{1}[\mathbf{k W}]$ & N/A & N/A \\
\hline$V_{2}[$ Volts] & 1.10 & $V_{1}+(0.01$ to 1.10$)$ \\
\hline $\begin{array}{l}P_{2}[\mathbf{k W}] \\
\text { (DERs that can only } \\
\text { generate active power) }\end{array}$ & $0.2 \cdot P_{\text {rated }}$ or $P_{\text {min }}$ & $P_{\text {min }}$ to $P_{\text {rated }}$ \\
\hline $\begin{array}{l}P_{2}[\mathbf{k W}] \\
\text { (DERs that can generate } \\
\text { and absorb active power) }\end{array}$ & 0 & $-P_{\text {rated }}$ to $+P_{\text {rated }}$ \\
\hline Response time [s] & 10 & 0.5 to 60 \\
\hline
\end{tabular}

must share with the DMS so that the Volt-Watt function could be effectively operationalized at the system level. They are:

- Nameplate information: Read-only information describing the as-built characteristics of each DER e.g., rated nameplate value for active power output from a DER $P_{\text {rated }}$.

- Configuration information: Read-Write parameters for the capacity and availability of each DER to perform the Volt-Watt function e.g., preset or configured minimum
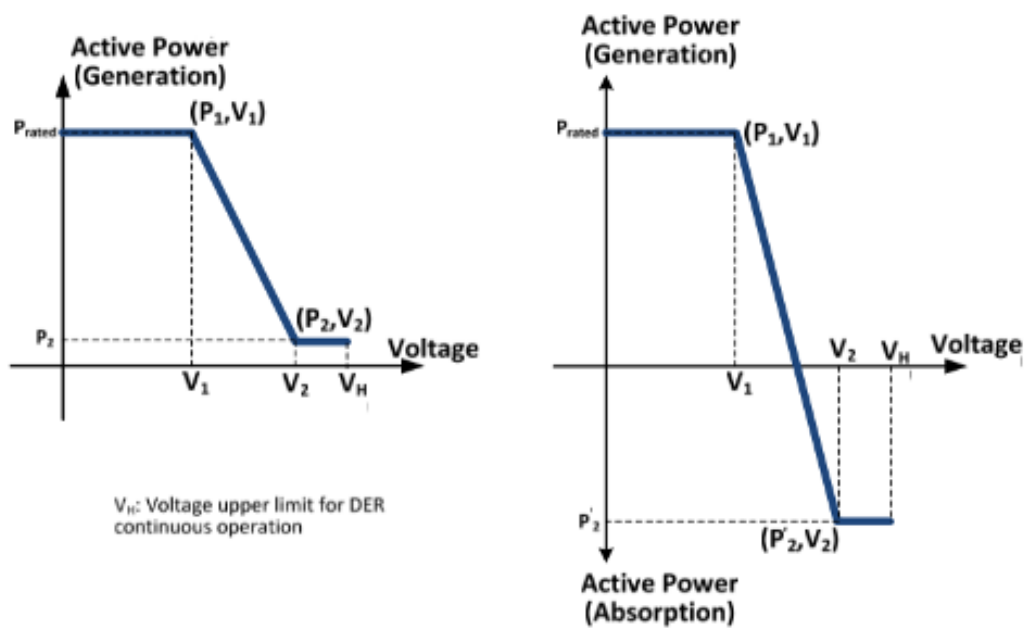

Fig. 25. Characteristic curves for Volt-Watt control of a DER as specified in the IEEE 1547 Standard. 


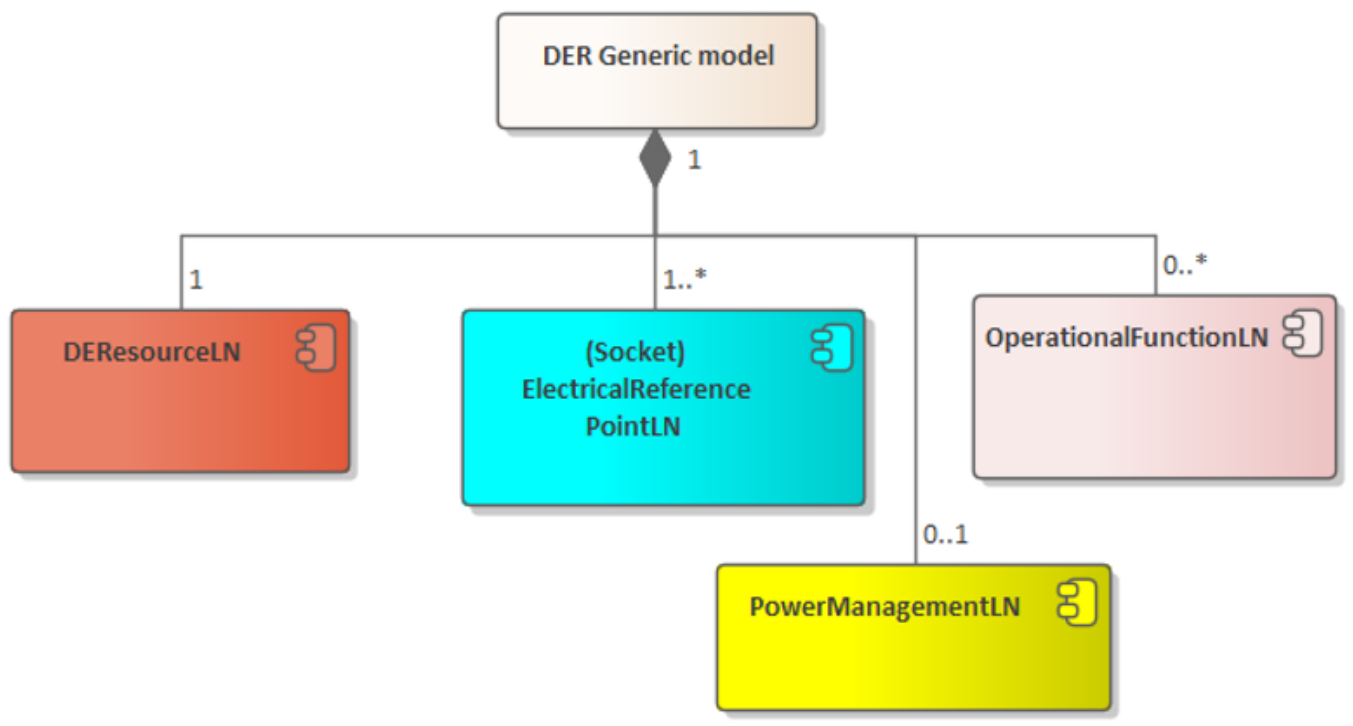

Fig. 26. Class model for a generic IEC 61850 DER showing the four major classes of asset information.

output power permitted for safe operation of a DER $P_{\text {min }}$.

- Monitoring information: Read-only measurements of the present operating conditions as reported by each DER e.g., the measured value of active power injection $P_{1}$.

- Management information: Read-Write mode change or mode activation information, including triggers to enable functions like the Volt-Watt response i.e., the grid operator defined set points for $V_{\text {nominal }}$.

These four classes of information together constitute a generic information model for a DER. Figure 26 illustrates the four information classes in the IEEE 1547 standard as logical nodes per the IEC 61850-7-420 object modeling specification. Organization of common data types and their classification in this manner provide several analytical and representational advantages, especially when modeling large systems.

A distributed generator, for example, is a sub-class of a generic DER, while also being a sub-class of a generic generator. In addition, it inherits objects from logical nodes, representing operational settings, system state, management information and name plate ratings. Hence, the derived class for a distributed generator (DGEN) can be expressed as a composition of several objects as illustrated in Figure 27.

Regarding the Volt-Watt use case for a distributed generator, IEEE 1547 parameters in Table 2 can be mapped to relevant operational settings, in already defined IEC 61850-7-420 
data objects ${ }^{9}$. Table 3 lists key parameter maps in configuration setting and nameplate information classes for the Volt-Watt behavior shown in Figure 25. This is not an exhaustive list and does not capture the real-time state or measurement data required for the Volt-Watt function.

Following the object-oriented architecture of IEC 61850, the resulting logical node for this function is a multi-inheritance sub-class of a distributed power management controller (DPMC).

The Volt-Watt example, chosen for illustration here, is decidedly straightforward; yet, implementation of this function could realistically require the real-time state of other DERs or measurements of nearby buses in the distribution grid. Given the wide variety of physical realizations of DER control schemes, it is advantageous to approach the DER controller as an abstract functional node, not physically bound to a particular DER, but rather associated to it via information flows.

Figure 28 illustrates a DER controller showing several communication pathways required to achieve reliable Volt-Watt functionality. Notably, measurements are drawn from grid coupling components such as circuit breakers and switches. Measurements are also drawn from the bus representing the point of coupling for the generator in question and the point of common coupling on the grid where the voltage is to be measured and regulated. The figure shows just one DER connected to a single bus in a circuit. However, as mentioned in this subsection, the information model for this distributed generator (DGEN) can be effectively scaled to a multi-generator system.

\subsection{Composition and Constraint Enforcement}

While consistency in information representation and data dependencies are crucial for composition, it is also necessary to consider the physical aspects of interoperability between DERs. In particular, if controllers for each DER share common state measurements from the grid, then deadlock conditions or parasitic effects may emerge from interactions between controllers.

One aspect of ensuring reliable co-operation between multiple DERs is to adhere to the constraints specified in the configuration settings in all operating conditions. Further, some safety critical functions may require constraint satisfaction guarantees on points of common coupling between multiple DERs.

Constraint enforcement of a composition of several subsystems can be achieved by enforcing individual constraints on each subsystem such that the net output of the combined system satisfies constraints. For example, consider the combined system:

$$
y(t)=y_{1}(t)+\cdots+y_{n}(t)
$$

subject to the constraint:

$$
y(t) \in \mathbb{Y}(t) \triangleq\{y(t): S y(t) \leq s\},
$$

${ }^{9}$ This mapping, along with mappings for seven other common DER functions, was provided by Christoph Brunner (ESTA International LLC) under contract from NIST. 
Table 3. A mapping between data objects in the IEC 61850-7-4 and IEC 61850-7-420 standard against parameters required for implementation of the IEEE 1547 Volt-Watt function.

\begin{tabular}{|c|c|c|}
\hline Parameter & Description & $\begin{array}{l}\text { IEC } 61850-7-4 / 420 \\
\text { Data Object }\end{array}$ \\
\hline $\begin{array}{l}\text { Active power rating at unity power factor } \\
\text { (nameplate active power rating) }\end{array}$ & Active power rating in Watts at unity power factor & $\begin{array}{l}\text { DGEN.WMaxRtg } \\
\text { DGEN.WMax }\end{array}$ \\
\hline $\begin{array}{l}\text { Active power rating at specified over- } \\
\text { excited power factor }\end{array}$ & $\begin{array}{l}\text { Active power rating in Watts at specified over- } \\
\text { excited power Factor }\end{array}$ & DGEN.WOvPFRtg \\
\hline Specified over-excited power factor & Over-excited power factor as described in 5.2 & DGEN.OvPFRtg \\
\hline $\begin{array}{l}\text { Active power rating at specified under- } \\
\text { excited power factor }\end{array}$ & $\begin{array}{l}\text { Active power rating in Watts at specified under- } \\
\text { excited power Factor }\end{array}$ & DGEN.WUnPFRtg \\
\hline Specified under-excited power factor & Under-excited power factor as described in 5.2 & DGEN.UnPFRtg \\
\hline Apparent power maximum rating & maximum apparent power rating in Volt-Amperes & $\begin{array}{l}\text { DGEN.VAMaxRtg } \\
\text { DGEN.VAMax }\end{array}$ \\
\hline Normal operating performance category & $\begin{array}{l}\text { indication of reactive power and voltage/power } \\
\text { control capability }\end{array}$ & DGEN.RegClas \\
\hline Reactive power injected maximum rating & Maximum injected reactive power rating in VArs & $\begin{array}{l}\text { DGEN.IvarMaxRtg } \\
\text { DGEN.IvarMax }\end{array}$ \\
\hline Reactive power absorbed maximum rating & Maximum absorbed reactive power rating in Vars & $\begin{array}{l}\text { DGEN.AvarMaxRtg } \\
\text { DGEN.AvarMax }\end{array}$ \\
\hline Active power charge maximum rating & Maximum active power charge rating in Watts & $\begin{array}{l}\text { DLOD.WMaxRtg } \\
\text { DLOD.WMax }\end{array}$ \\
\hline Apparent power charge maximum rating & $\begin{array}{l}\text { Maximum apparent power charge rating in Volt- } \\
\text { Amperes, may differ from the apparent power } \\
\text { maximum rating }\end{array}$ & $\begin{array}{l}\text { DLOD.VAMaxRtg } \\
\text { DLOD.VAMax }\end{array}$ \\
\hline AC voltage nominal rating & Nominal AC voltage rating in RMS Volts & DECP.EcpVRtg \\
\hline AC voltage maximum rating & Maximum AC voltage rating in RMS Volts & $\begin{array}{l}\text { DGEN.VMaxRtg } \\
\text { DGEN.VMax }\end{array}$ \\
\hline AC voltage minimum rating & Minimum AC voltage rating in RMS Volts & $\begin{array}{l}\text { DGEN.VMinRtg } \\
\text { DGEN.VMin }\end{array}$ \\
\hline Manufacturer & Manufacturer & LPHD.PhyNam.vendor \\
\hline Model & Model & LPHD.PhyNam.model \\
\hline Serial number & Serial number & LPHD.PhyNam.serNum \\
\hline Version & Version & $\begin{array}{l}\text { LPHD.PhyNam.hwRev } \\
\text { LPHD.PhyNam.swRev }\end{array}$ \\
\hline Limit active power enable & Enable mode & DWMX.FctEna \\
\hline Maximum active power & Maximum active power setting & DWMX.WLimPctSpt \\
\hline Voltage-active power mode enable & Enable voltage-active power mode & DVWC.FctEna \\
\hline V/P curve points & Voltage-active power curve points & DVWC.VWCrv \\
\hline Open loop response time & $\begin{array}{l}\text { Time to ramp up to } 90 \% \text { of the new active power } \\
\text { target in response to the change in voltage }\end{array}$ & DVWC.OplTmMax \\
\hline Constant power factor mode enable & Enable constant power factor mode & DEPF.FctEna \\
\hline Constant power factor & Constant power factor setting & DEPF.PFGnTgtSet \\
\hline Constant power factor excitation & & DEPF.PFGnExtSet \\
\hline HV trip curve points & High-voltage shall trip curve points & $\begin{array}{l}\text { Tr1PTOV.StrVal } \\
\text { Tr1PTOV.OpDITmms } \\
\text { Tr2PTOV.StrVal } \\
\text { Tr2PTOV.OpDITmms }\end{array}$ \\
\hline LV trip curve points & Low-voltage shall trip curve points & $\begin{array}{l}\text { Tr1PTOV.StrVal } \\
\text { Tr1PTOV.OpDITmms } \\
\text { Tr2PTOV.StrVal } \\
\text { Tr2PTOV.OpDITmms }\end{array}$ \\
\hline Limit active power enable & Enable mode & DWMX.FctEna \\
\hline Maximum active power & Maximum active power setting & DWMX.WLimPctSpt \\
\hline
\end{tabular}




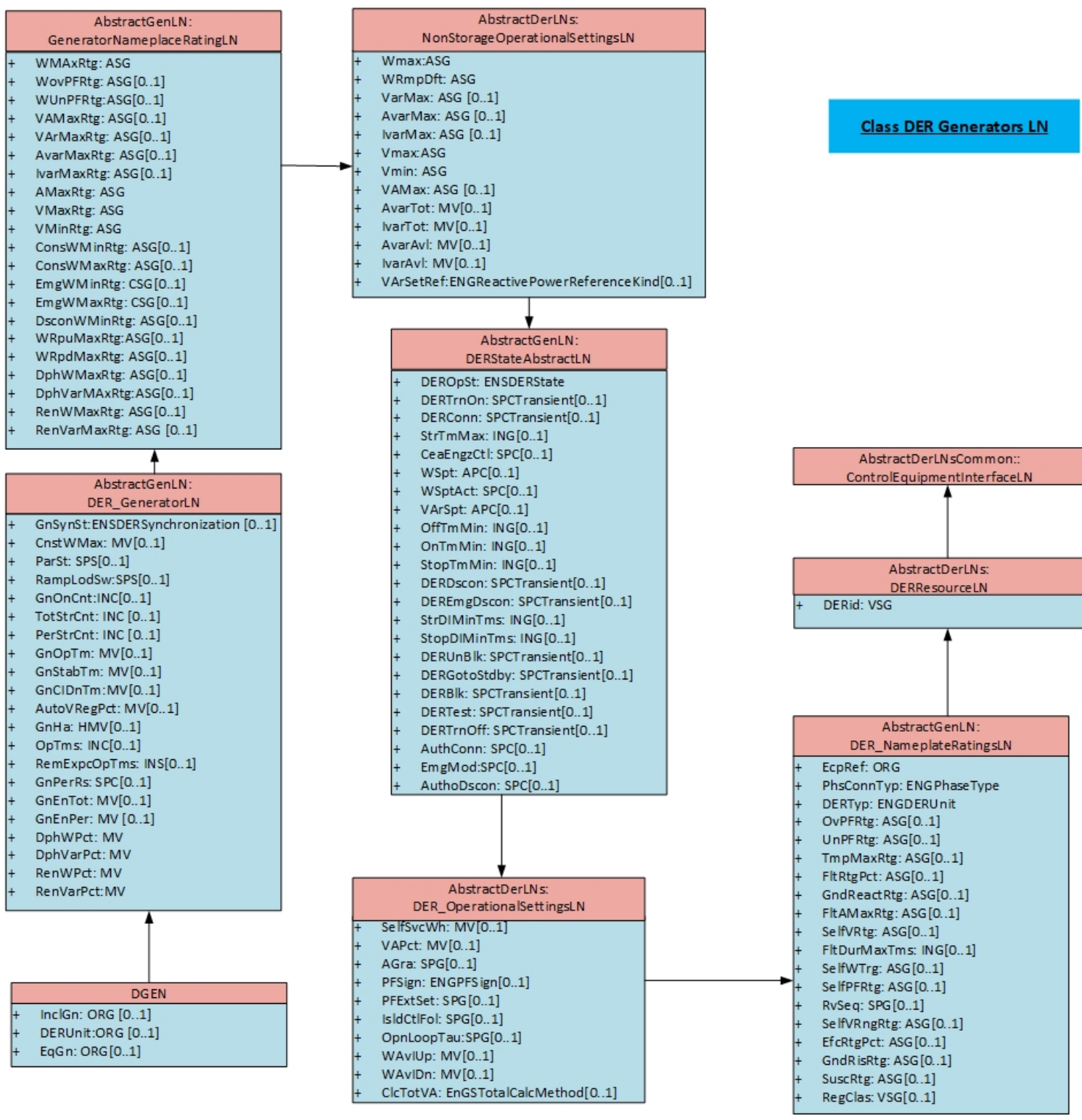

Fig. 27. Inheritance diagram for the IEC 61850-7-420 information model of a distributed generator (DGEN). 


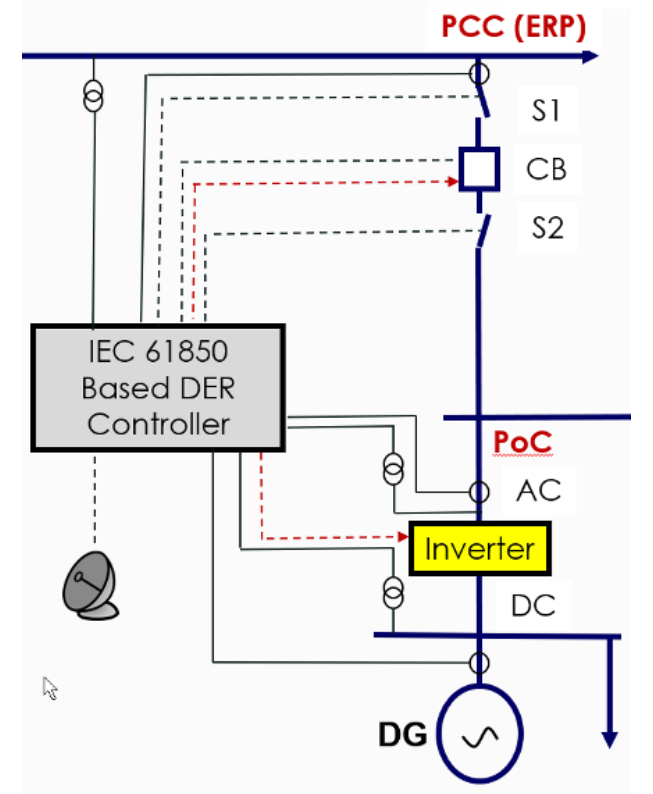

Fig. 28. A schematic diagram showing a generic DER controller and some of the several information flows that would be needed to implement core functions.

and let $y_{i}$ for all $i \in\{1, \ldots, n\}$ be the outputs of the subsystems given by:

$$
\begin{aligned}
x_{i}(t+1) & =A_{i} x_{i}(t)+B_{i} u_{i}(t) \\
y_{i}(t) & =C_{i} x_{i}(t)+D_{i} u_{i}(t)
\end{aligned}
$$

Substituting $y(t)$ from Eq. (2) into Eq. (3), yields:

$$
\left.y(t) \in \mathbb{Y}(t) \triangleq\left\{y(t): S y_{1}(t)+\cdots+S y_{n}(t)\right) \leq s\right\}
$$

which can be satisfied by individually enforcing

$$
y_{i}(t) \in \mathbb{Y}_{i}(t) \triangleq\left\{y_{i}(t): S y_{i}(t) \leq s_{i}\right\}
$$

where $s_{1}+\cdots+s_{n} \leq s$.

Constraint enforcement is integral to distributed DER control due to the tight power quality requirements on grid frequency, voltage, and power output. NIST is developing an Adaptive Reference Governor (RG) scheme to assist in distributed control of constrained DERs. The Reference Governor [85-95] is a control strategy that predicts the evolution of the system state to enforce pre-specified constraints on the inputs, states or the outputs of a system. The Reference Governor does this by modifying the reference to a pre-stabilized, closed-loop control system such that the system states and inputs belong to the Maximal Admissible Set (MAS). This MAS is defined as the set of all initial states and constant inputs that satisfy constraints for all future time. 


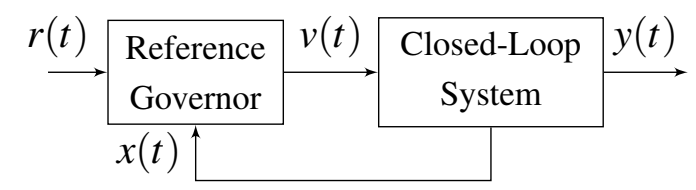

Fig. 29. Block diagram of RG governed system.

Because RG modifies the reference to a pre-stabilized closed-loop system, it can be described as an add-on scheme for constraint management. To illustrate this claim further, a block diagram of an RG-governed system is depicted in Figure 29. Note that the "closedloop system" is not modified in any way. However, an observer may be required to feedback predictions of the system states if they can't be measured.

As mentioned, the MAS of a system is defined as the set of all initial states and constant inputs such that the system's output constraints are satisfied for all future time. This is illustrated in Figure 29. In this case, the "closed-loop system", in general, can be described by the multi-input, multi-output, discrete-time, stable linear system:

$$
\begin{array}{r}
x(t+1)=A x(t)+B v(t) \\
y(t)=C x(t)+D v(t)
\end{array}
$$

The output $y(t)$ is subject to the following polyhedral constraints:

$$
y(t) \in \mathbb{Y} \triangleq\{y: S y \leq s\}
$$

Again, vector inequalities should be interpreted element-wise. In general, the set in (7) may be unbounded. RG employs MAS, denoted by $O_{\infty}$, which, to reiterate, is the set of all initial states and constant inputs that satisfy (7) for all time:

$$
O_{\infty}=\left\{(x, v): x(0)=x, v(t)=v, y(t) \in \mathbb{Y}, \forall t \in \mathbb{Z}^{+}\right\}
$$

As seen in (8), to construct MAS, $v(t)=v$ is held constant for all $t$. Using this assumption, the evolution of the output $y(t)$ can be expressed explicitly as a function of $x(0)=x$ and $v$ :

$$
y(t)=C A^{t} x+\left(C\left(I-A^{t}\right)(I-A)^{-1} B+D\right) v
$$

Therefore, MAS in (8) can be characterized by a polyhedron defined by an infinite number of inequalities:

$$
O_{\infty}=\left\{(x, v): S C A^{t} x+S\left(C\left(I-A^{t}\right)(I-A)^{-1} B+D\right) v \leq s, \forall t \in \mathbb{Z}^{+}\right\}
$$

It is shown in [96] that, under mild assumptions on $C$ and $A$, it is possible to determine this set finitely (i.e., describe as a finite number of inequalities) by constraining the steadystate value of $y$, denoted by $y(\infty)$, to the interior $\left(\varepsilon \in \mathbb{R}^{+}\right.$is a small number) of the constraint set:

$$
y(\infty) \triangleq\left(C(I-A)^{-1} B+D\right) v \in(1-\varepsilon) \mathbb{Y}
$$


Combining (10) and (11), an inner approximation of $O_{\infty}$ is obtained, denoted by the finite dimensional form $\widetilde{O}_{\infty}$, which can be represented by:

$$
\widetilde{O}_{\infty}=\left\{(x, v): H_{x} x+H_{v} v \leq h\right\}
$$

Where the matrices $H_{x}, H_{v}$, and $h$ are finite dimensional.

Note that MAS is calculated offline for use by RG online. During operation, RG utilizes the following update law to select a constraint-admissible modified reference $(v(t))$.

$$
v(t)=v(t-1)+\kappa(r(t)-v(t-1))
$$

Where $\kappa \in[0,1]$. To select $\kappa, \mathrm{RG}$ solves the following linear program in an effort to drive $v(t)$ as close to $r(t)$ as possible, without violating constraints:

$$
\begin{array}{ll}
\underset{\kappa \in[0,1]}{\operatorname{maximize}} & \kappa \\
\text { s.t. } & v(t)=v(t-1)+\kappa(r(t)-v(t-1)) \\
& (x(t), v(t)) \in \widetilde{O}_{\infty}
\end{array}
$$

Here, $x(t), r(t)$, and $v(t-1)$ are known parameters at time $t$. Note that (14) has an explicit solution due to the structure of the optimization problem's constraints. This makes RG numerically attractive, compared to alternative constraint management strategies, such as Model Predictive Control (MPC) [97-103] and Barrier Lyapunov Functions (BLF) [104106]. As such, RG is suitable for real-time control, especially that of fast processes, making it a viable framework for constraint management in distributed control of DERs.

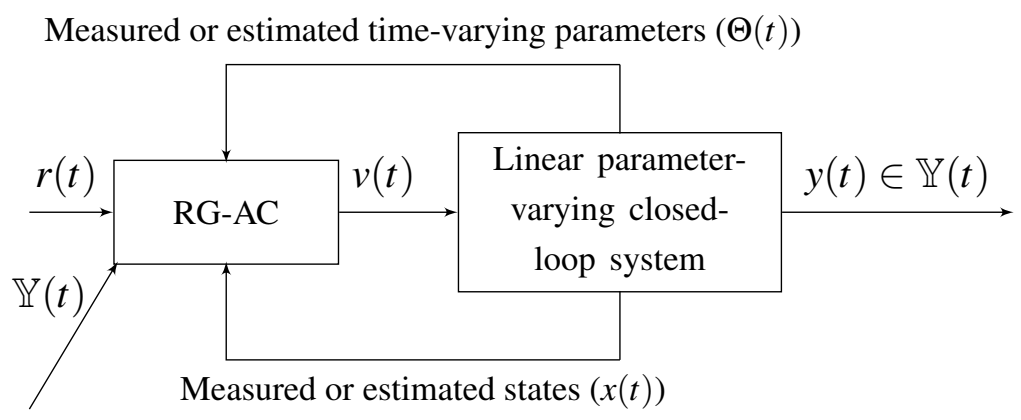

Fig. 30. Block diagram of RG-AC governed system.

NIST has been working to widen the capability of the RG scheme to addresses the constraints and dynamics of distribution circuits with significant DER penetration. Specifically, as Chapter 4 of [107] presents in detail, it is necessary for the RG to accommodate parameter-varying systems with time-varying constraints. NIST along with collaborating researchers have been developing an Adaptive-Contractive Reference Governor (RG-AC) which utilizes a contractive characterization of the Maximal Admissible Set that changes in real-time, as a function of the system's time-varying parameters, in a computationally 
attractive manner. A block diagram of an RG-AC-governed parameter-varying system is shown in Figure 30, where the "linear parameter-varying closed-loop system" is of the form:

$$
\begin{aligned}
x(t+1) & =A(\Theta(t)) x(t)+B(\Theta(t)) v(t) \\
y(t) & =C(\Theta(t)) x(t)
\end{aligned}
$$

Here, $\Theta(t) \in \mathbb{R}^{q}$ contains time-varying, scalar parameters on which the elements of $A, B$, and $C$ may depend. The reader is directed to [107] for a more detailed discussion of the RG-AC algorithm. Successful implementation of RG-AC requires the following implementation conditions:

- An accurate, discrete-time, model of the closed loop system in the form (15);

- the ability to measure or estimate the system states $(x(t))$ of $(15)$; and

- the ability to measure or estimate the time-varying parameters $(\Theta(t))$ of $(15)$.

In theory, the Adaptive-Contractive Reference Governor can be employed in DER control algorithms to handle the local constraints of individual DERs, while simultaneously enforcing constraints on the grid through distributed control methods. However, the three implementation conditions mentioned above have to be tested on practical circuits with realistic processing and communication constraints in order to establish feasibility of RGAC. This is the type of validation capability that the NIST campus power system is being instrumented to provide.

\subsection{Validation of the Information Exchange Required for DER Control}

The Adaptive-Contractive Reference Governor, as with other consensus or distributed control algorithms, can be analytically shown to guarantee performance only when constraints on computation time, measurement latency and state observability are assumed. These constraints are often difficult to realize in practice, which means that these control algorithms must be fully validated via simulation.

The validation approach often used by industry for complex systems, comprised of cyber and physical components, is real-time digital simulation (RTDS). Behavioral evaluation of physical hardware systems is achieved by solving model equations with real-world clock constraints on execution time [108, 109]. RTDS can also be used to perform hardware-inthe-loop (HIL) validation, where 'real' physical components are integrated with simulated components to improve confidence in test results. One example of a mature HIL validation process is where 'real' power conditioning equipment or power generating hardware is integrated with simulated controllers to create a power hardware-in-the-loop (PHIL) test system to aid in the rapid prototyping of controllers for DERs.

NIST has been leveraging internal expertise in electrical metrology, time transfer technology and test apparatus design to develop software libraries, calibration methods and automated test software. This is being done to meet the validation needs for the next generation of DER control systems [110]. 


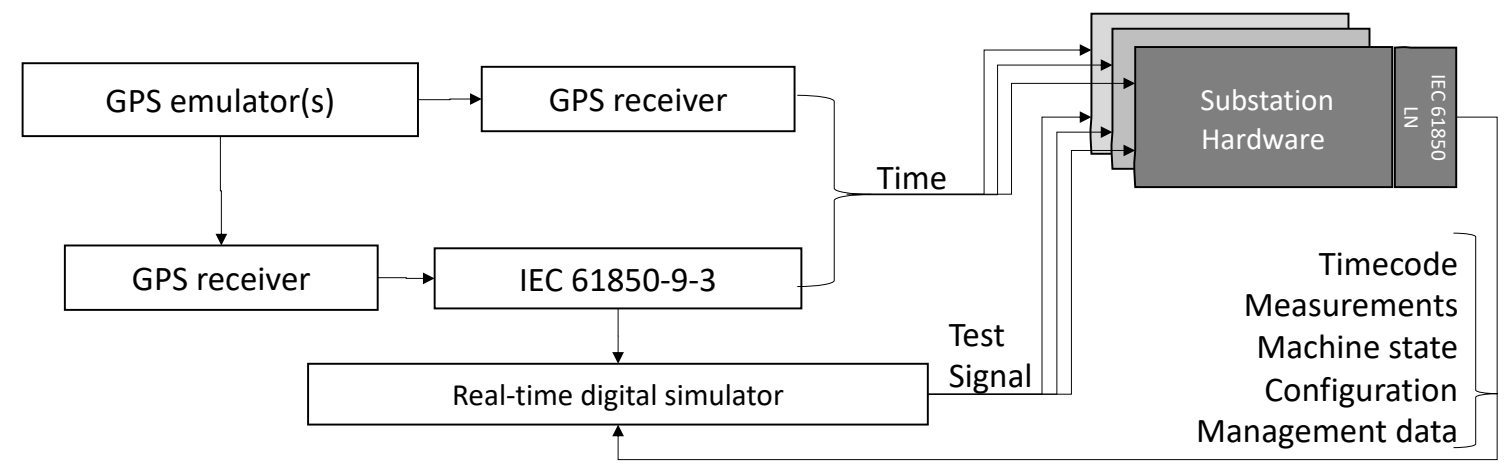

Fig. 31. A NIST developed hardware-in-the-loop test setup used to evaluate implementation of the IEC 61850-9-3 time transfer standard.

Figure 31 shows a test apparatus developed at NIST to evaluate protocol compliance and interoperability of time synchronization technologies included in the IEC 61850-8-3 specification. This test capability was part of a suite of NIST-developed interoperability test tools for an IEC 61850 plugfest [111].

Real substation hardware, complete with their IEC 61850 logical interfaces, were installed as HIL test articles. The test signals generated were voltage and current transients that would trigger discrete logic or circuit breaker action. Output data objects produced by every IEC 61850 LN was measured for accuracy using a closed-loop time measurement system implemented on the RTDS. The data types, parsed for evaluation, involved all the major classes, including streaming measurements, configuration settings and management information. Latency and jitter in these data were measured against a time reference timescale, established using a GPS (Global Positioning System) emulator and NISTcalibrated GPS receivers.

Other capabilities developed at NIST address gaps related to improving the robustness and repeatability of RTDS-based HIL validation. These capabilities include test methods to estimate the transfer function and uncertainty, resulting from the use of analog-to-digital and digital-to-analog interfaces for measuring and emulating proxy signals from/to power hardware. This capability uses components from NIST synchrometrology test apparatus (highlighted yellow in Figure 32(a)) along with the network test tools described above.

If $\beta \sin (\omega t+\phi)$ is assumed to be an emulation of the reference sinusoid $\alpha \sin (\omega t)$, then the associated error of a closed loop HIL system $\varepsilon(t)$ is also sinusoidal with a nominal gain $\gamma$. This gain can be rewritten as a function $\gamma^{*}$ of the induced phase delay $\phi$ as in (16). Figure 32(c) shows the emulation error magnitude $\varepsilon(t)$ for an HIL system, used for validating a DER against a frequency ramp condition. The data show that while the error magnitude observed align with $\gamma^{*}$, there are certain frequencies where the error magnitude is significantly attenuated. Non-uniformities in closed loop system response, such as presented here, present significant challenges to ensuring consistent test data from HIL systems. Accurately measuring these phenomena is the first step to compensating for them. 

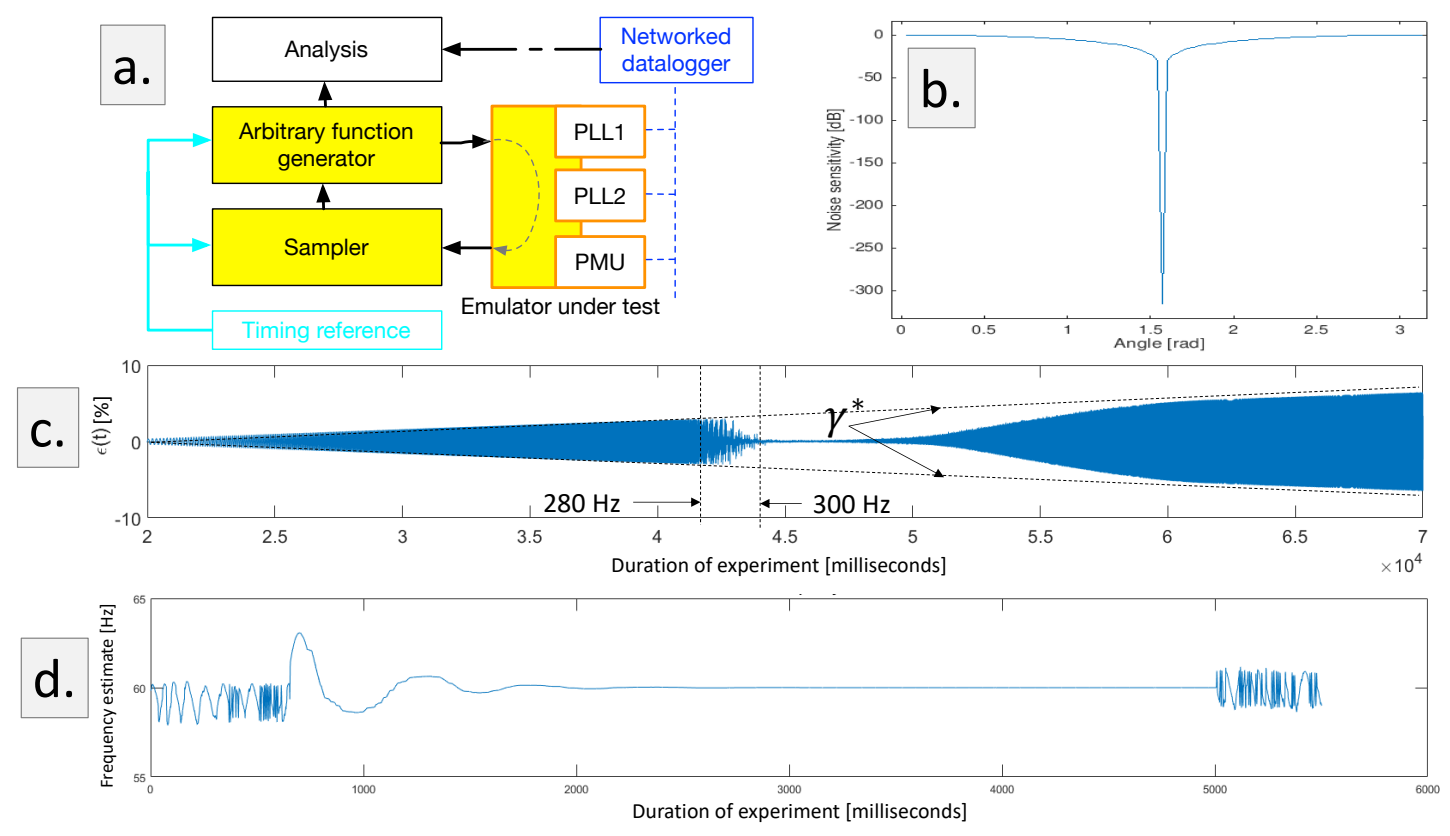

Fig. 32. Subfigure-a shows the calibration and compensation setup used to assess the performance of hardware-in-the-loop emulators. Subfigure-b shows the noise in an HIL system as function of phase error in the emulated signal. Subfigure-c shows non-uniformity in HIL system behavior where the measured error $\varepsilon(t)$ is deviates from $\gamma^{*}$ at specific frequencies. Subfigure-d shows gain dependent transient errors in the PLL based frequency estimates made by the emulator. 


$$
\begin{aligned}
\varepsilon(t): & =\alpha \sin (\omega t)-\beta \sin (\omega t+\phi)=\gamma \sin (\omega t+\delta) \\
\gamma & =\sqrt[2]{\alpha^{2}+\beta^{2}-\alpha \beta \cos (\phi)} \\
\gamma^{*} & =2 \sin \left(\frac{\phi}{2}\right)
\end{aligned}
$$

The test infrastructure developed for HIL validation can also be used to perform uncertainty analysis. For example, the expression for error magnitude in (16) can be extended to provide a measure of first order sensitivity, as a function of phase delay in an emulator. Figure 32(b) shows that the uncertainty in the system response is not uniform across all phase delays. In fact, the sensitivity of the system frequently has peaks or notches, as is the case here. This provides technical backing to anecdotal user observations of sustained mono-tonal oscillations in HIL systems in response to wide band excitation.

The NIST HIL test capability can also leverage calibration grade frequency estimation algorithms, used to assess phasor measurement units and frequency counters. Figure 32(d) shows transients in the frequency tracking loop of a commercial HIL emulator, in response to a "breaker close" event in the hardware being tested. The damped ringing behavior, shown in the figure, can be easily compensated for during a test. However, unless it is specifically characterized, this behavior would be indistinguishable from the transient response of the DER being tested. Also, it would compromise the reliability of any DER validation experiment.

\section{Acknowledgment}

This report captures the result of work done by multiple engineers and technical contributors. The authors would like to acknowledge the research contributions of Hamid Ossareh who was instrumental in the design of the reference governor. Sebastian Barillaro designed the software stack for the low-power wireless communication system and performed the early latency tests. Brian Dougherty was an invaluable resource for all things related to the measuring PV systems. Steve Bannon provided system administration and design support for the data server and the field server systems. Andrew Mundy designed the Layer-2 wireless network and provided key support in implementing the enterprise network security infrastructure. Terry Hahn maintains the wireless interface between the field servers and the NIST wireless network and performed the site survey for antenna placement and testing. Matthew Powell and the team at Also Energy Inc. developed the SCADA gateway server and provided support for the development of the Modbus TCP interface to the PV inverters. Tom Armstrong maintains the $5 \mathrm{MW}$ PV site and facilitated access and interactions during installation and testing. Norma Goldstein provided valuable editorial support. 


\section{References}

[1] Gopstein A, Nguyen C, O'Fallon C, Wollman D, Hasting N (2020) NIST Framework and Roadmap for Smart Grid Interoperability Standards, Release 4.0 (National Institute of Standards and Technology),

[2] Song EY, Nguyen C, Gopstein A (2019) Review of Smart Grid Standards for Testing and Certification Landscape Analysis. NIST Technical Note 2042 (US Department of Commerce, National Institute of Standards and Technology), .

[3] US Energy Information Administration (2011) Annual energy outlook 2011: With projections to 2035, Available at https://www.eia.gov/outlooks/aeo/pdf/0383(2011) .pdf.

[4] Povlsen AF (2002) Impacts of power penetration from photovoltaic power systems in distribution networks (International Energy Agency), IEA PVPS T5-10.

[5] Kashem MA, Ledwich G (2005) Multiple Distributed Generators for Distribution Feeder Voltage Support. IEEE Transactions on Energy Conversion 20(3):676-684.

[6] Woyte A, Van Thong V, Belmans R, Nijs J (2006) Voltage fluctuations on distribution level introduced by photovoltaic systems. IEEE Transactions on Energy Conversion 21(1):202-209.

[7] Paatero JV, Lund PD (2006) Effects of large-scale photovoltaic power integration on electricity distribution networks. Renewable Energy 32(2):216-234.

[8] Cardwell D (2015) Solar Power Battle Puts Hawaii at Forefront of Worldwide Changes. New York Times .

[9] Katiraei F, Sun C, Enayati B (2015) No Inverter Left Behind: Protection, Controls, and Testing for High Penetrations of PV Inverters on Distribution Systems. IEEE Power and Energy Magazine 13(2):43-49.

[10] Whitaker C, Newmiller J, Ropp M, Norris B (2008) Renewable Systems Interconnection Study: Distributed Photovoltaic Systems Design and Technology Requirements ,. SAND2008-0946 P.

[11] McDonald JD (2013) Solar Power Impacts Power Electronics In The Smart Grid. Power Electronics Magazine .

[12] Zeng Z, Yang H, Zhao R, Cheng C (2013) Topologies and control strategies of multifunctional grid-connected inverters for power quality enhancement: A comprehensive review. Renewable and Sustainable Energy Reviews 24:223-270.

[13] Shair J, Li H, Hu J, Xie X (2021) Power system stability issues, classifications and research prospects in the context of high-penetration of renewables and power electronics. Renewable and Sustainable Energy Reviews 145:111111.

[14] Gautam A, Veluvolu KC, Soh YC (2017) Communication-computation tradeoff in distributed consensus optimization for mpc-based coordinated control under wireless communications. Journal of the Franklin Institute 354(9):3654-3677.

[15] Liu Y, Cheng S, Ning B, Li Y (2019) Performance enhancement using durational model predictive control combined with backstepping control and disturbance observer for electrical drives. Journal of Vibration and Control 25(4):946-959. 
[16] Zhou S (2021) The effect of smart meter penetration on dynamic electricity pricing: Evidence from the united states. The Electricity Journal 34(3):106919.

[17] Lovins AB (2017) Reliably integrating variable renewables: Moving grid flexibility resources from models to results. The Electricity Journal 30(10):58-63.

[18] Campbell RJ (2015) Cybersecurity issues for the bulk power system.

[19] Ghosh AK, Lubkeman DL, Downey MJ, Jones RH (1997) Distribution circuit state estimation using a probabilistic approach. IEEE Transactions on Power Systems 12(1):45-51.

[20] Steinbrink C, Lehnhoff S (2016) Quantifying probabilistic uncertainty in smart grid co-simulation. 2016 Workshop on Modeling and Simulation of Cyber-Physical Energy Systems (MSCPES), , pp 1-6.

[21] Zhu Q, Başar T (2011) Robust and resilient control design for cyber-physical systems with an application to power systems. 2011 50th IEEE Conference on Decision and Control and European Control Conference, , pp 4066-4071.

[22] Li J, Ou X, Rajagopalan R (2010) Uncertainty and risk management in cyber situational awareness. Cyber Situational Awareness :51-68.

[23] Abur A, Exposito AG (2004) Power system state estimation: theory and implementation (CRC press), .

[24] Rohjans S, Uslar M, Bleiker R, González J, Specht M, Suding T, Weidelt T (2010) Survey of smart grid standardization studies and recommendations. 2010 First IEEE International Conference on Smart Grid Communications, , pp 583-588.

[25] McLaren J (2014) Advanced Inverter Function to Support High Levels of Distributed Solar (NREL, Golden, CO), BR-6A20-62612.

[26] Basso T (2014) IEEE 1547 and 2030 Standards for Distributed Energy Resources Interconnection and Interoperability with the Electricity Grid (NREL), NREL/TP5D00-63157.

[27] Fan Z, Kulkarni P, Gormus S, Efthymiou C, Kalogridis G, Sooriyabandara M, Zhu Z, Lambotharan S, Chin WH (2013) Smart grid communications: Overview of research challenges, solutions, and standardization activities. IEEE Communications Surveys Tutorials 15(1):21-38.

[28] Almassalkhi M, Hiskens IA (2013) Temperature-based model-predictive cascade mitigation in electric power systems. Proceedings of the Conference on Decision and Control (IEEE), , pp 7560-7567.

[29] Almassalkhi MR, Hiskens IA (2015) Model-Predictive Cascade Mitigation in Electric Power Systems With Storage and Renewables-Part I: Theory and Implementation. IEEE Transactions on Power Systems 30(1):67-77.

[30] Almassalkhi M, Hiskens IA (2014) Model-Predictive Cascade Mitigation in Electric Power Systems With Storage and Renewables, Part II: Case-Study. IEEE Transactions on Power Systems 30(1):78-87.

[31] Hermans RM, Almassalkhi M, Hiskens IA (2012) Incentive-based Coordinated Charging Control of Plug-in Electric Vehicles at the Distribution-Transformer Level. IEEE American Control Conference :264-269. 
[32] Goebel R, Sanfelice RG, Teel AR (2009) Hybrid dynamical systems. IEEE Control Systems Magazine 29(2):28-93.

[33] Van Der Schaft AJ, Schumacher JM (2000) An introduction to hybrid dynamical systems. Lecture Notes in Control and Information Sciences, Vol. 251 (SpringerVerlag, London), .

[34] Sauer PW, Behera AK, Pai MA, Winkelman JR, Chow JH (1989) Trajectory approximations for direct energy methods that use sustained faults with detailed power system models. IEEE Transactions on Power Systems 4(2):499-506.

[35] Tian MW, Shi CJR (2000) Worst case tolerance analysis of linear analog circuits using sensitivity bands. IEEE Transactions on Circuits and Systems I: Fundamental Theory and Applications 47(8):1138-1145.

[36] Hiskens IA, Pai MA (2000) Trajectory sensitivity analysis of hybrid systems. IEEE Transactions on Circuits and Systems I: Fundamental Theory and Applications 47(2):204-220.

[37] Hiskens IA, Pai MA, Nguyen TB (2000) Bounding uncertainty in power system dynamic simulations. IEEE Power Engineering Society Winter Meeting 2:1533-1537.

[38] Hiskens IA (2001) Nonlinear dynamic model evaluation from disturbance measurements. IEEE Transactions on Power Systems 16(4):702-710.

[39] Zima M, Korba P, Andersson G (2003) Power systems voltage emergency control approach using trajectory sensitivities. IEEE Conference on Control Applications 1:189-194 vol.1.

[40] Hiskens IA, Alseddiqui J (2006) Sensitivity, Approximation, and Uncertainty in Power System Dynamic Simulation. IEEE Transactions on Power Systems 21(4):1808-1820.

[41] Althoff M, Krogh BH, Stursberg O (2011) Analyzing reachability of linear dynamic systems with parametric uncertainties. Modeling, Design, and Simulation of Systems with Uncertainties, eds Rauh A, Auer E (Springer), , pp 69-94.

[42] Asarin E, Dang T, Frehse G, Girard A, Le Guernic C, Maler O (2006) Recent progress in continuous and hybrid reachability analysis. IEEE Conference on Computer Aided Control Systems Design (Munich, Germany), , pp 1582-1587.

[43] Platzer A, Clarke EM (2007) The image computation problem in hybrid systems model checking. Hybrid Systems: Computation and Control :473-486.

[44] Kurzhanski AB, Varaiya P (2000) Ellipsoidal techniques for reachability analysis: internal approximation. System and Control Letters 41:201-211.

[45] Kurzhanski AB, Varaiya P (2002) On ellipsoidal techniques for reachability analysis. Part I: external approximations. Optimization methods and software 17(2):177-206.

[46] Hafner MR, Del Vecchio D (2009) Computation of safety control for uncertain piecewise continuous systems on a partial order. IEEE Conference on Decision and Control, , pp 1671-1677.

[47] Botchkarev O, Tripakis S (2000) Verification of hybrid systems with linear differential inclusions using ellipsoidal approximations. International Workshop on Hybrid Systems: Computation and Control (Springer), , pp 73-88. 
[48] Jin L, Kumar R, Elia N (2010) Reachability analysis based transient stability design in power systems. Journal of Electrical Power and Energy Systems 32:782-787.

[49] Chen YC, Dominguez-Garcia AD (2011) Assessing the Impact of Wind Variability on Power System Small-Signal Reachability. Hawaii International Conference on System Sciences, , pp 1-8.

[50] Pico HNV, Aliprantis DC, Hoff EC (2013) Reachability analysis of power system frequency dynamics with new high-capacity HVAC and HVDC transmission lines. Bulk Power System Dynamics and Control:1-9.

[51] Dhople SV, Chen YC, Dominguez-Garcia AD (2013) A Set-Theoretic Method for Parametric Uncertainty Analysis in Markov Reliability and Reward Models. IEEE Transactions on Reliability 62(3):658-669.

[52] Girard A (2005) Reachability of uncertain linear systems using zonotopes. Hybrid Systems: Computation and Control, eds Morari M, Thiele L (Springer Berlin Heidelberg), Lecture Notes in Computer Science, Vol. 3414, pp 291-305.

[53] Girard A, Le Guernic C (2008) Zonotope/hyperplane intersection for hybrid systems reachability analysis. Hybrid Systems: Computation and Control, eds Egerstedt M, Mishra B (Springer Berlin Heidelberg), Lecture Notes in Computer Science, Vol. 4981, pp 215-228.

[54] Althoff M, Krogh BH (2014) Reachability analysis of nonlinear differentialalgebraic systems. IEEE Transactions on Automatic Control 59(2):371-383.

[55] Althoff M (2014) Formal and compositional analysis of power systems using reachable sets. IEEE Transactions on Power Systems 29(5):2270-2280.

[56] Fan J, Fan H, Backscheider P, McDonald JD (2012) Coordinated optimization in distribution operation. IEEE Power and Energy Society General Meeting, , pp 1-5.

[57] Mills-Price M, Hao K (2014) The Importance of Coordinated Control Systems in Solar Generation Plants (Schweitzer Engineering Laboratories, Inc.), TP6658-01.

[58] Turitsyn K, Sulc P, Backhaus S, Chertkov M (2010) Distributed control of reactive power flow in a radial distribution circuit with high photovoltaic penetration. IEEE Power and Energy Society General Meeting :1-6.

[59] Kundu S, Backhaus S, Hiskens IA (2013) Distributed control of reactive power from photovoltaic inverters. IEEE Interational Symposium on Circuits and Systems (Beijing), , pp 249-252.

[60] Zhang B, Lam AYS, Dominguez-Garcia AD, Tse D (2014) An Optimal and Distributed Method for Voltage Regulation in Power Distribution Systems. IEEE Transactions on Power Systems .

[61] Bolognani S, Carli R, Cavraro G, Zampieri S (2014) Distributed reactive power feedback control for voltage regulation and loss minimization. IEEE Transactions on Automatic Control 60(4):966-981.

[62] Nedic A, Olshevsky A, Ozdaglar A, Tsitsiklis JN (2009) On distributed averaging algorithms and quantization effects. IEEE Transactions on Automatic Control 54(11):2506-2517.

[63] Bolognani S, Zampieri S (2013) A distributed control strategy for reactive power 
compensation in smart microgrids. IEEE Transactions on Automatic Control 58(11):2818-2833.

[64] Meng L, Zhao X, Tang F, Savaghebi M, Dragicevic T, Vasquez JC, Guerrero JM (2015) Distributed voltage unbalance compensation in islanded microgrids by using a dynamic consensus algorithm. IEEE Transactions on Power Electronics 31(1):827-838.

[65] Dörfler F, Simpson-Porco JW, Bullo F (2014) Plug-and-play control and optimization in microgrids. IEEE Conference on Decision and Control, , pp 211-216.

[66] Dörfler F, Simpson-Porco J, Bullo F (2014) Breaking the Hierarchy: Distributed Control \& Economic Optimality in Microgrids. arXivorg .

[67] Simpson-Porco JW, Dörfler F, Bullo F (2012) Droop-controlled inverters in microgrids are Kuramoto oscillators. IFAC Workshop on Distributed Estimation and Control in Networked Systems (Citeseer), , pp 264-269.

[68] Simpson-Porco JW, Dörfler F, Bullo F (2013) Synchronization and power sharing for droop-controlled inverters in islanded microgrids. Automatica 49(9):2603-2611.

[69] Adhikari S, Li F (2014) Coordinated V-f and P-Q Control of Solar Photovoltaic Generators With MPPT and Battery Storage in Microgrids. IEEE Transactions on Smart Grid 5(3):1270-1281.

[70] IEEE (2003) IEEE standard for interconnecting distributed resources with electric power systems. IEEE Std 1547-2003:1-28.

[71] Commission IIE, et al. (2017) Iec ts 62786: Distributed energy resources connection with the grid. IEC Std.

[72] IEEE (2011) Guide for smart grid interoperability of energy technology and information technology operation with the electric power system, end-use applications, and loads. IEEE Std 2030-2011:1-126.

[73] Johnson J, Gonzalez S, Ralph ME, Ellis A, Broderick R (2013) Test Protocols for Advanced Inverter Interoperability Functions (Sandia National Lab),

[74] Prabakar K, Singh A, Tombari C (2019) IEEE 1547-2018 based interoperable PV inverter with advanced grid-support functions. 46th Photovoltaic Specialists Conference (PVSC) (IEEE), , pp 2072-2077.

[75] Schneider KP, Chen Y, Chassin DP, Pratt RG, Engel DW, Thompson S (2008) Modern grid initiative: Distribution taxonomy final report (PNNL),

[76] Schneider KP, Fuller JC, Tuffner FK, Singh R (2010) Evaluation of Conservation Voltage Reduction (CVR) on a National Level (PNNL), PNNL-19596.

[77] Nazir N, Racherla P, Almassalkhi M (2020) Optimal multi-period dispatch of distributed energy resources in unbalanced distribution feeders. IEEE Transactions on Power Systems 35(4):2683-2692.

[78] Narkhede N, Shapira G, Palino T (2017) Kafka: the definitive guide: real-time data and stream processing at scale ("O'Reilly Media, Inc."), .

[79] Merkel D (2014) Docker: lightweight linux containers for consistent development and deployment. Linux journal 2014(239):2.

[80] de Brito MAG, Sampaio LP, Luigi G, e Melo GA, Canesin CA (2011) Comparative 
analysis of mppt techniques for pv applications. 2011 International Conference on Clean Electrical Power (ICCEP), , pp 99-104.

[81] Brunner C (2008) IEC 61850 for power system communication. 2008 IEEE/PES Transmission and Distribution Conference and Exposition, , pp 1-6.

[82] Ustun TS, Ozansoy C, Zayegh A (2011) Distributed energy resources (DER) object modeling with iec 61850-7-420. AUPEC 2011, , pp 1-6.

[83] Mackiewicz RE (2006) Overview of IEC 61850 and benefits. 2006 IEEE Power Engineering Society General Meeting, , pp 1-8.

[84] Photovoltaics DG, Storage E (2018) Ieee standard for interconnection and interoperability of distributed energy resources with associated electric power systems interfaces. IEEE Std :1547-2018.

[85] Kolmanovsky I, Garone E, Di Cairano S (2014) Reference and command governors: A tutorial on their theory and automotive applications. 2014 American Control Conference (IEEE), , pp 226-241.

[86] Gilbert EG, Kolmanovsky I (1995) Discrete-time reference governors for systems with state and control constraints and disturbance inputs. Proc. IEEE Conference on Decision and Control, Vol. 2, pp 1189-1194.

[87] Garone E, Di Cairano S, Kolmanovsky I (2017) Reference and command governors for systems with constraints: A survey on theory and applications. Automatica 75:306-328.

[88] Gilbert EG, Kolmanovsky I (1999) Fast reference governors for systems with state and control constraints and disturbance inputs. International Journal of Robust and Nonlinear Control: IFAC-Affiliated Journal 9(15):1117-1141.

[89] Kolmanovsky IV, Gilbert EG (1995) Maximal output admissible sets for discretetime systems with disturbance inputs. Proc. American Control Conference (IEEE), Vol. 3, pp 1995-1999.

[90] Bemporad A (1998) Reference governor for constrained nonlinear systems. IEEE Transactions on Automatic Control 43(3):415-419.

[91] Ossareh HR (2019) Reference governors and maximal output admissible sets for linear periodic systems. International Journal of Control :1-13.

[92] Osorio J, Ossareh HR (2018) A stochastic approach to maximal output admissible sets and reference governors. Proc. IEEE Conference on Control Technology and Applications, , pp 704-709.

[93] Laracy A, Ossareh H (2020) Constraint management for batch processes using iterative learning control and reference governors. Proceedings of Machine Learning Research (PMLR), , p 1-10.

[94] Osorio J, Santillo M, Seeds JB, Jankovic M, Ossareh HR (2019) A reference governor approach towards recovery from constraint violation. 2019 American Control Conference (ACC) (IEEE), , pp 1779-1785.

[95] Liu Y, Osorio J, et al. (2018) Decoupled reference governors for multi-input multioutput systems. 2018 IEEE Conference on Decision and Control (CDC) (IEEE), , pp 1839-1846. 
[96] Gilbert EG, Tan KT (1991) Linear systems with state and control constraints: the theory and application of maximal output admissible sets. IEEE Transactions on Automatic Control 36(9):1008-1020.

[97] Maciejowski JM (2008) Predictive control: with constraints (Prentice Hall), .

[98] Camacho EF, Alba CB (2013) Model predictive control (Springer Science \& Business Media), .

[99] Goodwin G, Seron MM, De Doná JA (2006) Constrained control and estimation: an optimisation approach (Springer Science \& Business Media), .

[100] Kwon WH, Han SH (2006) Receding horizon control: model predictive control for state models (Springer Science \& Business Media), .

[101] Morari M, Lee JH (1999) Model predictive control: past, present and future. Computers \& Chemical Engineering 23(4-5):667-682.

[102] Mayne D, Rawlings J, Rao C, Scokaert P (2000) Constrained model predictive control: Stability and optimality. Automatica 36(6):789 - 814.

[103] Wang Y, Boyd S (2010) Fast model predictive control using online optimization. IEEE Transactions on Control Systems Technology 18(2):267-278.

[104] Tee K, Ge S, Tay E (2009) Barrier lyapunov functions for the control of outputconstrained nonlinear systems. Automatica 45(4):918 - 927.

[105] Romdlony MZ, Jayawardhana B (2016) Stabilization with guaranteed safety using control lyapunov-barrier function. Automatica 66:39 - 47.

[106] Tee KP, Ge SS (2011) Control of nonlinear systems with partial state constraints using a barrier lyapunov function. International Journal of Control 84(12):20082023.

[107] Freiheit C (2020) Reference Governors for Time-varying Systems and Constraints Master's thesis University of Vermont.

[108] Menghal PM, Laxmi AJ (2012) Real time simulation: Recent progress challenges. 2012 International Conference on Power, Signals, Controls and Computation, , pp $1-6$.

[109] Omar Faruque MD, et al. (2015) Real-time simulation technologies for power systems design, testing, and analysis. IEEE Power and Energy Technology Systems Journal 2(2):63-73.

[110] Lundstrom B, Shirazi M, Coddington M, Kroposki B (2013) An advanced platform for development and evaluation of grid interconnection systems using hardware-inthe-loop: Part III-grid interconnection system evaluator. NREL Technical Report 5500-57516.

[111] Anand DM, Brady KG, Song Y, Nguyen CT, Lee KB, FitzPatrick GJ, Goldstein AR, Li-Baboud Y (2019) NIST smart grid interoperability test tools. NIST Special Publication 1900-801. 